\title{
Production of high brightness $\gamma$ rays through backscattering of laser photons on high-energy electrons
}

\author{
I. Sakai, ${ }^{1}$ T. Aoki,${ }^{2}$ K. Dobashi, ${ }^{1, *}$ M. Fukuda, ${ }^{1}$ A. Higurashi, ${ }^{2, \dagger}$ T. Hirose,${ }^{1}$ T. Iimura, ${ }^{3}$ Y. Kurihara, ${ }^{4}$ T. Okugi, ${ }^{4}$ \\ T. Omori, ${ }^{4}$ J. Urakawa, ${ }^{4}$ M. Washio, ${ }^{2}$ and K. Yokoya ${ }^{4}$ \\ ${ }^{1}$ Department of Physics, Tokyo Metropolitan University, 1-1 Minamiohsawa, Hachioji-shi, Tokyo 192-0397, Japan \\ ${ }^{2}$ Advanced Research Institute for Science and Engineering, Waseda University, 3-4-1 Okubo, Shinjuku-ku, Tokyo 169-8555, Japan \\ ${ }^{3}$ Faculty of Engineering, Seikei University, 3-3-1, Kichijoji-Kitamachi, Musashino-shi, Tokyo 180-8633, Japan \\ ${ }^{4}$ KEK: High Energy Accelerator Research Organization, 1-1 Oho, Tsukuba-shi, Ibaraki 305-0801, Japan \\ (Received 15 January 2002; revised manuscript received 26 February 2003; published 9 September 2003)
}

Based on the requirements from a conceptual design of a polarized positron beam for future linear colliders, we constructed a special collision system with a short focal length of $150 \mathrm{~mm}$ of the laser beams so as to produce $\gamma$ rays through inverse Compton scattering. In order to achieve efficient laserelectron collisions, we created a special optics to produce very small $e^{-}$-beam sizes of $\sigma_{e_{x 0}}=7.6 \mu \mathrm{m}$ and $\sigma_{e_{y 0}}=5.4 \mu \mathrm{m}$ in the horizontal and vertical directions at the collision point. Using laser light with a wavelength of $532 \mathrm{~nm}$ and an $e^{-}$beam of $1.28 \mathrm{GeV}$, provided from the ATF-damping ring at KEK, we generated $2 \times 10^{5} \gamma$ rays with a time duration of $26 \mathrm{ps}$ in rms, leading to a peak brightness of $1.7 \times$ $10^{18} /\left(\mathrm{mrad}^{2} \mathrm{~mm}^{2} 0.1 \%\right.$ bandwidth $\left.\mathrm{s}\right)$ near to the maximum energy of $56 \mathrm{MeV}$.

DOI: 10.1103/PhysRevSTAB.6.091001

PACS numbers: 52.38.Ph, 42.60.Jf, 42.62.-b, 41.75.Ht

\section{INTRODUCTION}

It is widely accepted that one of the next-generation accelerators at the high-energy frontier will be an electron $\left(e^{-}\right)$-positron $\left(e^{+}\right)$linear collider (LC) which plays a role complementary to the hadron collider LHC coming into operation in 2007 at CERN. Specific features of a LC are to allow the acceleration of polarized $e^{-}$and $e^{+}$ beams without depolarization. In high-energy regimes of around a few hundred $\mathrm{GeV}$, the helicity can be interpreted as the chirality, which is one of the important quantities in field theory.

The key points of discovering exotic phenomena at the JLC $[1,2]$ are to efficiently suppress dominant contributions from the ordinary reaction processes predicted by the standard model, e.g., $W$ pair production, $e^{-} e^{+} \rightarrow$ $W^{-} W^{+}$. Actually, around the center-of-mass energy at $500 \mathrm{GeV}$, the cross section of $W$ pair production is almost 2 orders higher than those of interesting but rare processes, such as supersymmetric particle production, which we want to observe [1]. Fortunately, in accordance with the standard model, $W$ pair production occurs dominantly through the combination of $e_{L}^{-} e_{R}^{+}$, with suffices $R$ and $L$ representing the right- and left-handed helicities. Hence, if we employ $e^{-}$beams with right-handed helicity, we can considerably suppress the contribution of $W$ pair production. However, owing to the fact that the magnitude of the $e^{-}$polarization is not $100 \%$, we necessarily suffer contamination caused by processes which are induced by the left-handed component of the $e^{-}$ helicity [3] and are prevented from clearly observing

\footnotetext{
*Present address: National Institute of Radiological Science.

${ }^{\dagger}$ Present address: Fuji Photo Optical Co., Ltd.
}

rare events. If a positron is polarized in addition to an electron, we can significantly increase the effective polarization, defined as

$$
P_{\text {eff }}=\frac{P_{1}-P_{2}}{1-P_{1} P_{2}}
$$

where $P_{1}$ and $P_{2}$ stand for the magnitudes of the polarizations of the $e^{-}$and $e^{+}$beams, respectively [4]; negative values of $P_{1}$ and $P_{2}$ correspond to the left-handed polarization. Any error in $P_{\text {eff }}$ can be reduced considerably if both the positrons and electrons are polarized [5]. We also mention particular roles of transverse polarization as follows [6]. Since a transversely polarized state is a linear combination of longitudinally polarized states, new physics can be observed through interference between the initial states, which have different combinations of longitudinal polarizations, such as $e_{R}^{-} e_{R}^{+}$and $e_{L}^{-} e_{R}^{+}$. To realize transverse polarization, both beams must be polarized. Consequently, the controllability of the spins of both the electrons and positrons will bring further possibilities for LC to extract small and significant signals by using the spin-dependent characteristics of the reaction processes.

In 1995, we proposed, as illustrated in Fig. 1, a new method in which highly polarized positrons can be generated via two fundamental processes, namely, the Compton scattering of circularly polarized laser-light off relativistic electrons and successive $e^{-}-e^{+}$pair creation [4,7]. Since then, proof-of-principle experiments have been conducted at KEK using an $e^{-}$beam of $1.28 \mathrm{GeV}$ and laser light with a wavelength of $532 \mathrm{~nm}$, generated from the second harmonic of a Nd:YAG laser. In the first-stage experiment, we developed a basic technique for creating positrons using a linearly polarized 


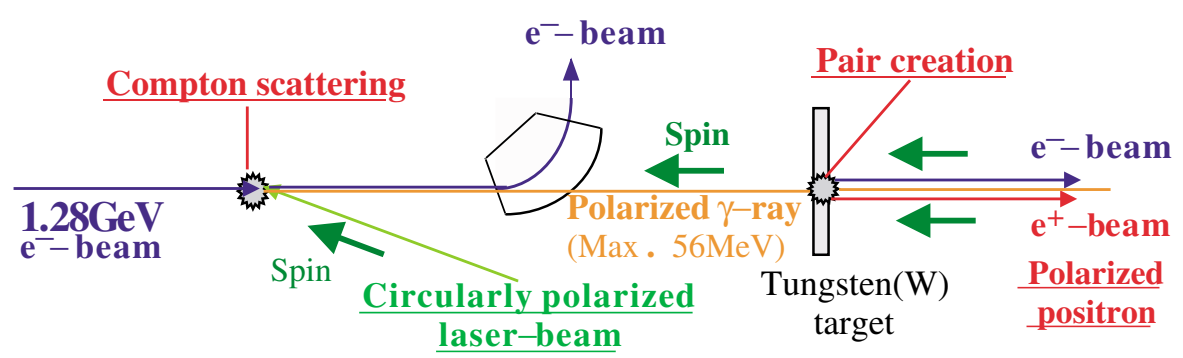

FIG. 1. (Color) Concept of polarized positron generation. The circularly polarized laser beam is backscattered on the electron beam and generates polarized $\gamma$ rays, which successively pair create polarized positrons.

laser beam, and in 1998 the first demonstration to generate pair-created positrons was successfully achieved based on our proposed scheme [8,9].

The JLC requires high-quality $e^{+}\left(e^{-}\right)$beams with considerably high intensity, i.e., $0.7 \times 10^{10} e^{+} /$bunch, as well as extremely small emittances of $\epsilon_{e_{x}}=$ $7.7 \times 10^{-10} \mathrm{radm}$ and $\epsilon_{e_{y}}=7.7 \times 10^{-12} \mathrm{radm}$ at $1.98 \mathrm{GeV}[1,2]$. Hence, we should conduct detailed experimental studies to achieve highly efficient and stable collisions of laser beams with relativistic $e^{-}$beams. For creating $\gamma$ rays with the required intensity of $3.5 \times$ $10^{11} \gamma \mathrm{s} /$ bunch, our conceptual design of the JLC polarized $e^{+}$beam demands that, as illustrated in Refs. $[9,10]$, $50 \mathrm{CO}_{2}$ laser pulses of a picosecond time duration consecutively collide with one $e^{-}$bunch with an energy of $5.8 \mathrm{GeV}$, a population of $5 \times 10^{10} e^{-} \mathrm{s} /$ bunch, and a normalized emittance of $5 \times 10^{-6} \mathrm{rad}$. Because the 50 collision points are installed within a limited distance of $4.8 \mathrm{~m}$, we must establish a special colliding system with a short focal length of laser beams.

In order to accomplish the production of high-intensity $\gamma$ rays, we have been pursuing the study of two essential technical points. Namely, first we will bring a high-power $\mathrm{CO}_{2}$ laser into practical use to generate high-intensity photons, as required by the conceptual design. Second, we should achieve the optimized conditions for $e^{-}$and laser beams to realize an efficient collision. The Japan/U.S. cooperation program in high-energy physics started in 1998 in collaboration with the group at the Brookhaven Accelerator Test Facility (BNL-ATF), which has been developing a high-power $\mathrm{CO}_{2}$ laser. In 1999 using a newly constructed collision chamber, named "Compton chamber," in which we attained the short focal length of $150 \mathrm{~mm}$ for the laser beam, we produced high-intensity $\mathrm{x}$ rays of $3 \times 10^{7}$ photons/pulse with a pulse width of 3.5 ps using the $60 \mathrm{MeV}$ linac and $0.6 \mathrm{GW} \mathrm{CO}_{2}$ [11,12]. For studying the other crucial problem of accomplishing efficient $e^{-}$-laser collisions, we have developed the polarized positron source at the KEK Accelerator Test Facility called the KEK-ATF provided for investigating various technical aspects needed for the JLC. The KEK experiment permits us to do a more realistic study with respect to the collision system, $\gamma$-ray detectors, $\gamma$-ray polarimetry, and so on because the generated $\gamma$ rays at the current experiment have almost the same maximum energy of about $60 \mathrm{MeV}$ as those required by the conceptual design of the JLC polarized positron source $[9,10]$. We have therefore conducted a $\gamma$-ray production experiment using particular $e^{-}$beam optics. We have also developed a numerical model in which we take into account various properties of both the $e^{-}$and laser beams, i.e., the emittances, and the space and time distributions.

We describe the $e^{-}$-beam optics and beam-diagnostics system of the extracted $e^{-}$-beam line of the KEK-ATF damping ring in Sec. II A as well as the laser system, including a Compton chamber with a short focal length in Sec. II B. The experimental results of $\gamma$-ray productions are given in Sec. IIC. A numerical model is described in Sec. III and compared with data to clarify detailed properties of collisions. Section IV is devoted to conclusions and future prospects. We also address evaluation of the present method in terms of applicability for the JLC polarized positron source.

\section{EXPERIMENTS}

\section{A. Electron-beam optics}

The KEK-ATF damping ring, which provides an $e^{-}$ beam of $1.28 \mathrm{GeV}$ with a repetition of $1.56 \mathrm{~Hz}$, has been operated at the KEK for detailed investigations on the properties of the extremely high-quality $e^{-}$beams required from the JLC. First of all, the coordinates are defined as shown in Fig. 2. The origin is the collision point of an electron and a laser photon, the $s$ axis is parallel to the $e^{-}$-beam line, and the $x$ and $y$ axes are the horizontal and vertical ones. In 1998, it was demonstrated that the KEK damping ring has the smallest horizontal emittance in the world [13]. Ordinary values of the horizontal and vertical emittances are $\epsilon_{e_{x}}=(1.7 \pm$ $0.3) \times 10^{-9} \mathrm{rad} \mathrm{m}$ and $\epsilon_{e_{y}}=(1.5 \pm 0.75) \times 10^{-11} \mathrm{rad} \mathrm{m}$, respectively [14]. The present experiment was carried out on the extraction line of an $e^{-}$beam designed especially for measuring a small emittance. Figure 3 shows the extraction line, which is equipped with bending $(B)$ and quadrupole $(Q)$ magnets for controlling the beam optics.

As described in Sec. II B, the focal length of the laser beam is designed to be $150 \mathrm{~mm}$, which leads to a laser 


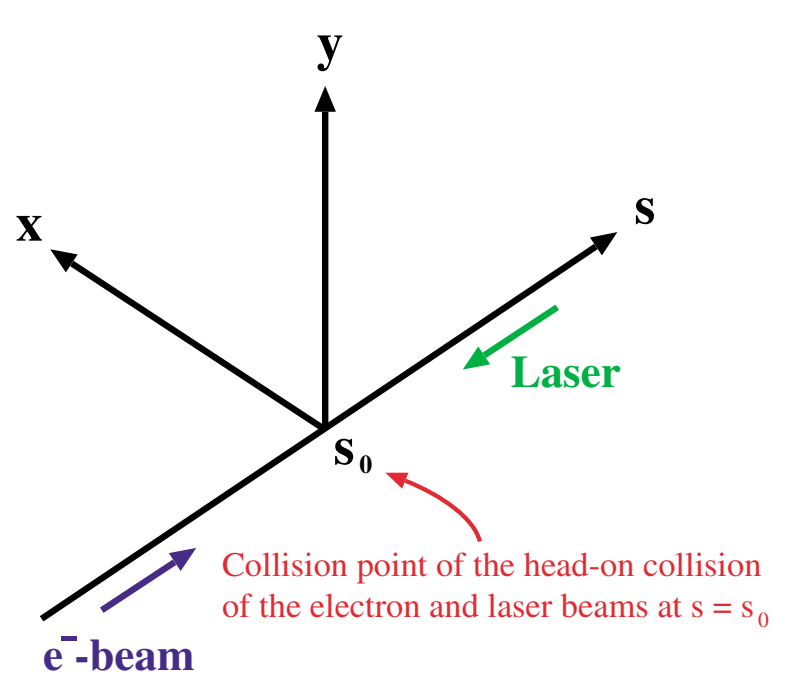

FIG. 2. (Color) Coordinates.

spot size of approximately $6 \mu \mathrm{m}$ in rms at the collision point. For the realization of effective collisions, the electron-beam size should be close to that of the laser spot size. Because the optics of the extraction line, called "the normal optics" hereafter, is utilized to measure the small emittance, the dispersion is designed to be zero at the positions of the wire scanners, and thus the $e^{-}$-beam size at the collision point becomes large, such as $\sim 500 \mu \mathrm{m}$ for the horizontal direction and $10-30 \mu \mathrm{m}$ for the vertical direction. We therefore have to create a different optics, called "the collision optics," which is suitable for our collision experiments. We thus took the following steps for finalizing the collision optics: first, we examined various optics in which the horizontal beam size should be considerably reduced, and then we found an efficient and practical method to realize the designed beam size.

Based on a numerical model, whose details are discussed in Sec. IV, we calculated the luminosity of electron-laser collisions as a function of the horizontal $e^{-}$-beam size $\left(\sigma_{e_{x 0}}\right)$ at the collision point under the assumptions that the laser spot size $\left(\sigma_{\ell_{x 0}}=\sigma_{\ell_{y 0}}\right)$ is $6 \mu \mathrm{m}$ and the vertical $e^{-}$-beam size $\left(\sigma_{e_{v 0}}\right)$ is $6 \mu \mathrm{m}$. The expected number of generated $\gamma$ rays $\left(N_{\gamma}\right)$ is shown in Fig. 4.

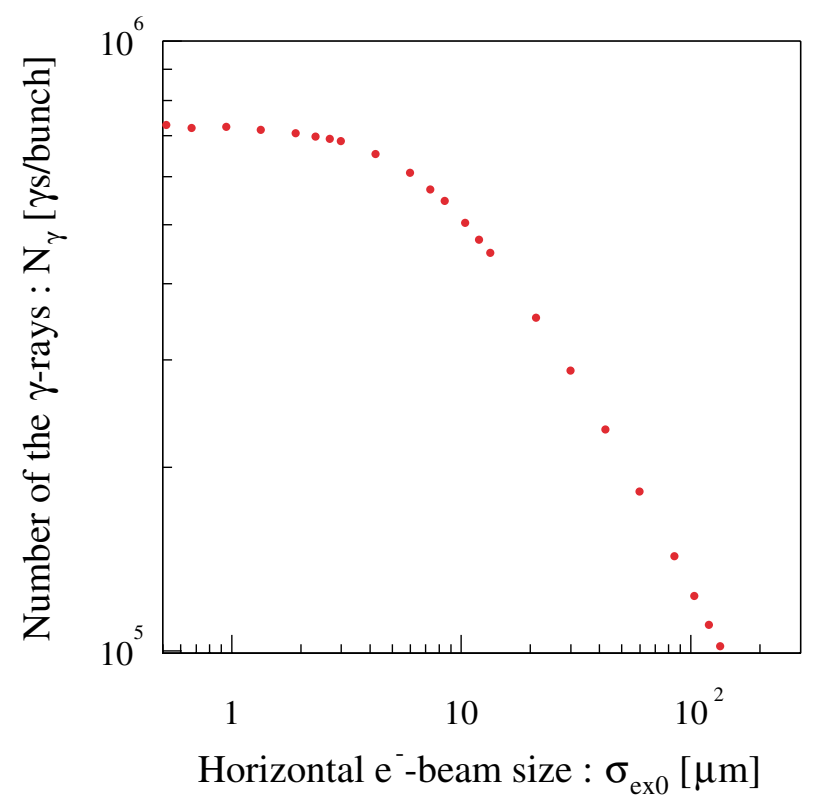

FIG. 4. (Color) Expected number of $\gamma$ rays as a function of the horizontal $e^{-}$-beam size calculated by the numerical model.

As $\sigma_{e_{x 0}}$ decreases towards $\sigma_{e_{x 0}}=3 \mu \mathrm{m}, N_{\gamma_{\text {cal }}}$ increases. Therefore, it is desirable to make the $e^{-}$-beam size close to $3 \mu \mathrm{m}$.

The $e^{-}$-beam size at any position $(s)$ along the beam trajectory can be represented as

$$
\sigma_{e}(s)=\sqrt{\epsilon_{e} \beta_{e}(s)+\eta^{2}(s)\left(\frac{\sigma_{p}}{p}\right)^{2}}
$$

where $\epsilon_{e}$ is the emittance, $\sigma_{p} / p$ the momentum spread, $\beta_{e}(s)$ the beta function, and $\eta(s)$ the dispersion function. In designing the collision optics, we studied the precise behaviors of the $e^{-}$beam in terms of the functions $\beta_{e}(s)$ and $\eta(s)$ using the beam optics code SAD (Strategic Accelerator Design) [15]. As is mentioned in Sec. II B, a screen monitor inside the Compton chamber has insufficient resolution to measure small $e^{-}$and laser beams. Thus, it was used just for the alignment purpose.

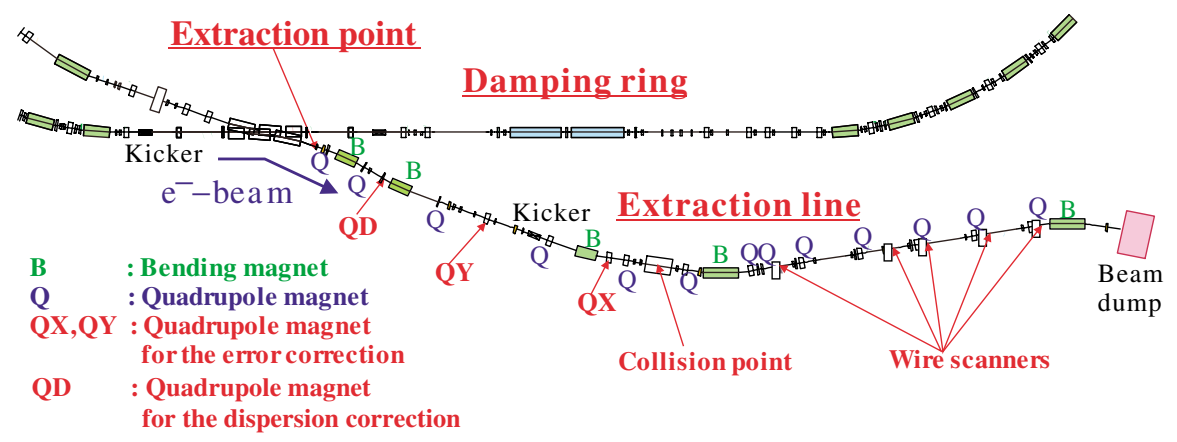

FIG. 3. (Color) Extraction beam line of the damping ring. Bending and quadrupole magnets are indicated. 
A procedure to determine the collision optics is given as follows: (i) The momentum spread $\left(\sigma_{p} / p\right)$ is obtained by measuring the beam size with a screen monitor being located at a position with high dispersion. (ii) Under the normal optics for which $\eta$ is designed to be small in a region where wire scanners are equipped, we measure the beam sizes using five wire scanners and determine the emittances $\left(\epsilon_{e_{x}}, \epsilon_{e_{y}}\right)$ and Twiss parameters at the extraction point. (iii) Using the thus determined parameters, we look for the collision optics which is optimized with respect to the beam size at the collision point $s=s_{0}$ as well as the sensitivity of the optics to errors in the magnetic fields of the $Q$ magnets. It is noted that because $\sigma_{p} / p$ and $\epsilon_{e}$ are determined by the operation conditions of the damping ring, the parameters should be measured for each run. Figure 5 depicts the simulated horizontal beam size $\sigma_{e_{x 0}}$ as a function of $\beta_{e_{x 0}}$ at the collision point $s_{0}$. The emittance used is given in Table I and the momentum spread is assumed to be zero. Note that $\sigma_{e_{x 0}}$ becomes small as $\beta_{e_{x 0}}$ decreases in the region $\beta_{e_{x 0}}>$ 0.01 . The chromaticity $[\xi(s)]$ is defined as

$$
\xi\left(s_{0}\right)=\frac{1}{4 \pi} \int_{s_{e x}}^{s_{0}} K\left(s^{\prime}\right) \beta\left(s^{\prime}\right) d s^{\prime},
$$

where the parameter $K(s)$ represents the strength of the $Q$-magnet's focusing and $\mathrm{S}_{\mathrm{ex}}$ is the value at the extraction point. As shown in Fig. $6, \xi(s)$ increases rapidly in the region $\beta_{e_{x 0}} \leq 0.03$, leading to a large $\sigma_{e_{x 0}}$. Therefore, the optimum value for $\beta_{e_{x 0}}$ is 0.03 that corresponds to the design beam size $\sigma_{e_{x 0}}=7.1 \mu \mathrm{m}$. Owing to the extremely small value of the vertical emittance, $\epsilon_{e_{v}}=1.5 \times$ $10^{-11} \mathrm{rad} \mathrm{m}$, the vertical beam size $\left(\sigma_{e_{y 0}}\right)$ is obtained

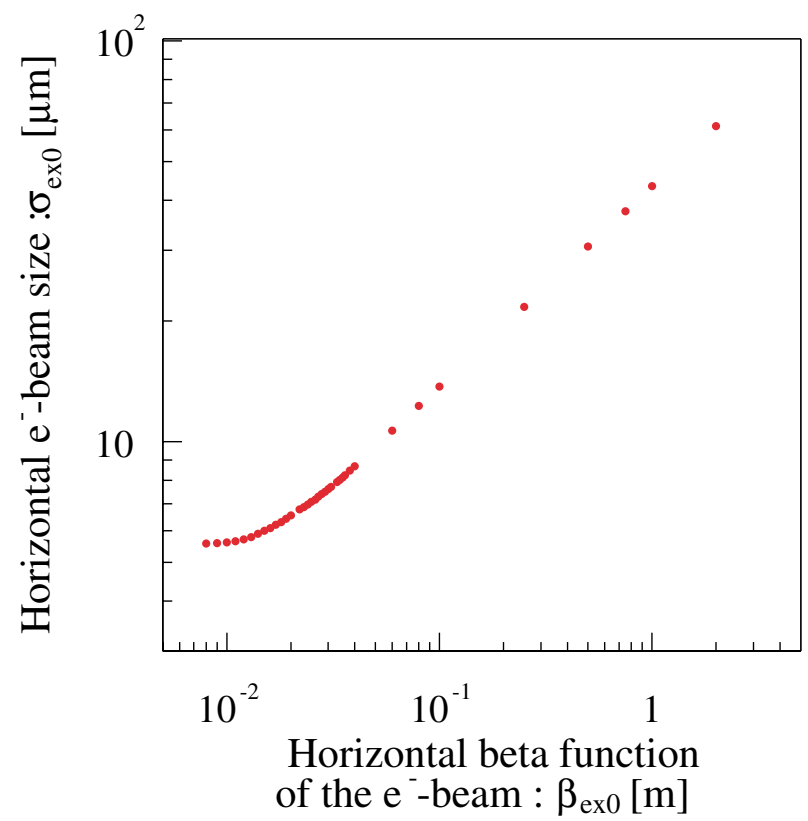

FIG. 5. (Color) Simulated horizontal $e^{-}$-beam size as a function of the horizontal beta function using SAD.
TABLE I. Parameters of the $e^{-}$and laser beams.

\begin{tabular}{|c|c|c|}
\hline \multicolumn{3}{|l|}{ Parameter } \\
\hline Electron beam & & Measured parameters \\
\hline Energy (GeV) & $E$ & 1.28 \\
\hline Population ( $e^{-} /$bunch $)$ & $N_{e}$ & $(0.90 \pm 0.04) \times 10^{10}$ \\
\hline \multicolumn{3}{|l|}{ Emittance $(\mathrm{rad} \mathrm{m})$} \\
\hline Horizontal & $\epsilon_{e_{x}}$ & $1.76 \times 10^{-9}$ \\
\hline Vertical & $\epsilon_{e_{y}}$ & $1.50 \times 10^{-11}$ \\
\hline \multicolumn{3}{|l|}{ Dispersion function (m) } \\
\hline Horizontal & $\eta_{x}$ & -0.0036 \\
\hline Vertical & $\eta_{y}$ & -0.0011 \\
\hline Momentum spread & $\frac{\sigma_{p}^{p}}{p}$ & $7.7 \times 10^{-4}$ \\
\hline Bunch length (ps) & $\tau_{e}$ & 26 \\
\hline \multicolumn{3}{|l|}{ Position jitter $(\mu \mathrm{m})$} \\
\hline Horizontal & $\Delta_{e_{x}}$ & 3.2 \\
\hline Vertical & $\Delta_{e_{y}}$ & 2.2 \\
\hline \multicolumn{3}{|l|}{ Angle jitter $(\mu \mathrm{rad})$} \\
\hline Horizontal & $\theta_{e_{x}}$ & 68.50 \\
\hline \multirow[t]{2}{*}{ Vertical } & $\theta_{e_{y}}$ & 48.67 \\
\hline & & Designed parameters \\
\hline \multicolumn{3}{|l|}{ Beta function (m) } \\
\hline Horizontal & $\beta_{e_{x 0}}$ & 0.03 \\
\hline \multirow[t]{2}{*}{ Vertical } & $\beta_{e_{y 0}}$ & 2.00 \\
\hline & & Estimated parameters \\
\hline \multicolumn{3}{|l|}{ Beam size $(\mu \mathrm{m})$} \\
\hline Horizontal & $\sigma_{e_{x 0}}$ & $7.6 \pm 0.5$ \\
\hline Vertical & $\sigma_{e_{y 0}}$ & $5.4 \pm 0.6$ \\
\hline Laser & & Measured parameters \\
\hline Energy (mJ/pulse) & $E$ & 370 \\
\hline \multicolumn{3}{|l|}{ Emittance (rad m) } \\
\hline Horizontal & $\epsilon_{\ell_{x}}$ & $(15.6 \pm 0.2) \times 10^{-8}$ \\
\hline Vertical & $\epsilon_{\ell_{y}}$ & $(13.9 \pm 0.2) \times 10^{-8}$ \\
\hline \multicolumn{3}{|l|}{ Position jitter $(\mu \mathrm{m})$} \\
\hline Horizontal & $\Delta_{\ell_{x}}$ & 1.2 \\
\hline Vertical & $\Delta_{\ell_{y}}$ & 3.2 \\
\hline \multirow[t]{2}{*}{ Pulse width (ns) } & $\tau_{\ell}$ & $3.6 \pm 0.1$ \\
\hline & & Designed parameters \\
\hline Wavelength (nm) & $\lambda$ & 532 \\
\hline \multirow[t]{2}{*}{ Focal length (mm) } & $f$ & 150 \\
\hline & & Estimated parameters \\
\hline \multicolumn{3}{|l|}{ Spot size $(\mu \mathrm{m})$} \\
\hline Horizontal & $\sigma_{\ell_{x 0}}$ & $6.0 \pm 0.2$ \\
\hline Vertical & $\sigma_{\ell_{y 0}}$ & $5.3 \pm 0.2$ \\
\hline
\end{tabular}

as $\sqrt{\epsilon_{e_{y}} \beta_{e_{y 0}}}=5.5 \mu \mathrm{m}$. Figure 7 shows the variation in the various parameters along the extracted $e^{-}$-beam line in the collision optics designed for the present experiment. The horizontal axis shows position $s$ together with $Q$ magnets and the collision point. Since in actual cases there are errors in the magnetic field of each $Q$ magnet, deviations of the beam size from the designed values take place. Hence, in order to obtain the minimum beam sizes, readjustments of the magnetic fields should usually be 


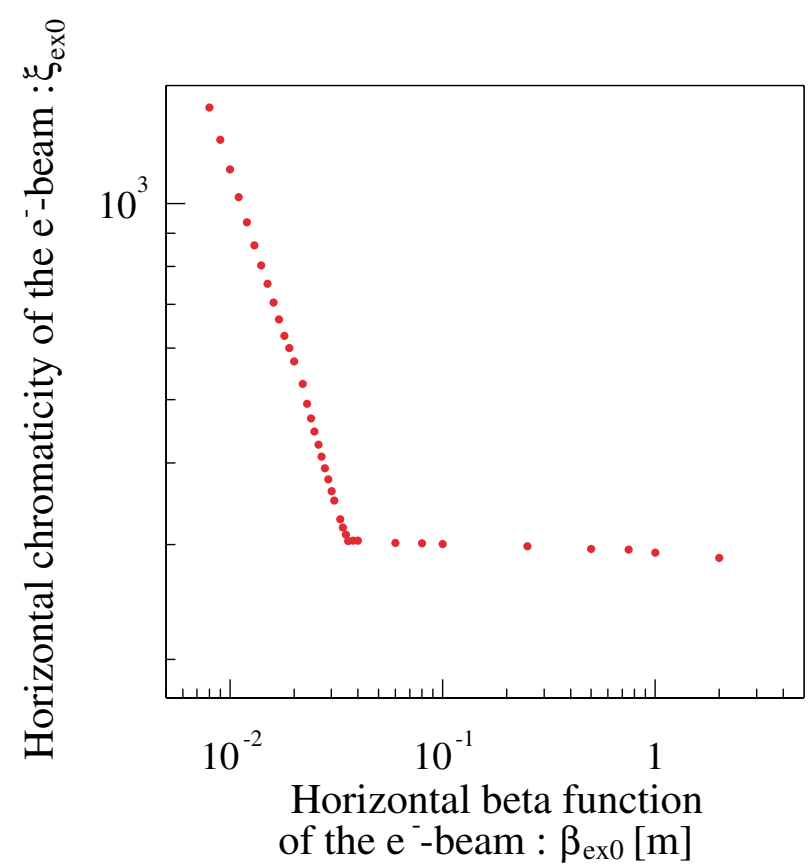

FIG. 6. (Color) Horizontal chromaticity of the $e^{-}$beam as a function of the horizontal beta function calculated using SAD.

made for the eight $Q$ magnets installed upstream before the collision point. Using SAD, we examined the effects of errors on the beam size under the assumption that the $Q$ magnets have random errors of currents less than $0.5 \mathrm{~A}$, fluctuating around the designed values. Each point in Fig. 8(a) represents the beam sizes $\left(\sigma_{e_{x 0}}\right.$ and $\left.\sigma_{e_{v 0}}\right)$ at the collision point, the spread of which is considerably larger than the designed values. At this stage, the dispersion may be large because the calculated beam sizes are considerably large as compared with the designed values, for which the dispersion is assumed to be zero. Having extensively studied the relation between the magnetic fields of each $Q$ magnet and the beam size, we have revealed that the errors in the vertical beam size caused by the eight $Q$ magnets could be compensated by adjusting the current of only one magnet $(Q Y)$, which only little affects

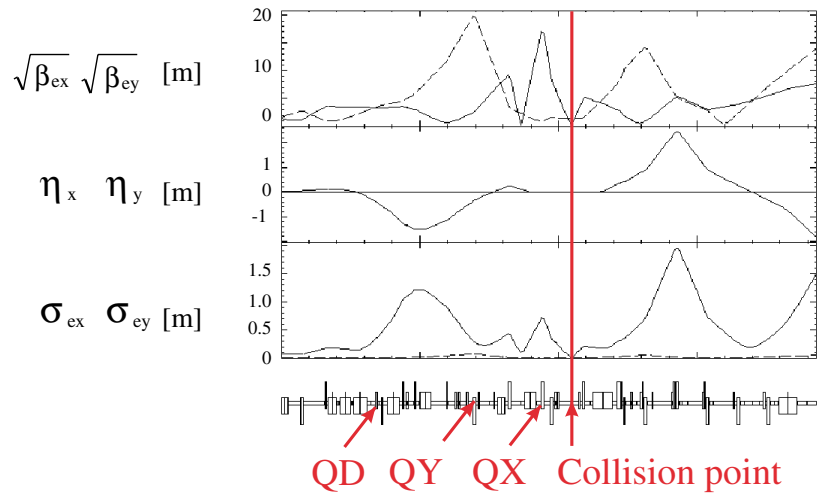

FIG. 7. (Color) Designed optics calculated by SAD. the horizontal beam size. By varying the current of $Q Y$, we searched in the SAD for the minimum value of the vertical beam size, as shown in Fig. 8(b). In a similar manner, it was found that only one $Q$ magnet $Q X$ absorbed the errors of the horizontal beam size. Consequently, we determined the minimum beam size in both horizontal and vertical directions, as depicted in Fig. 8(c). Through the course of Figs. 8(a)-8(c), we have attempted only to reduce the beam size, leaving the magnitude of the dispersion arbitrary. We therefore adjusted another $Q$ magnet $Q D$ so as to reduce the dispersion without having any significant effect on the beam size and obtained the beam-size distribution shown in Fig. 8(d).

By observing the relative beam size with the screen monitors, we also reaffirmed experimentally that the $Q$ magnets $(Q X, Q Y)$ could effectively compensate all errors of the horizontal and vertical beam sizes caused by the eight $Q$ magnets. By changing the current of $Q X$ and $Q Y$, we could clearly observe the minimum points at $I_{x}=$ 71.21 A and $I_{y}=25.39 \mathrm{~A}$, as shown in Figs. 9(a) and 9(b). Fixing the current of $Q X$ and $Q Y$ to be $I_{x}$ and $I_{y}$, we measured the dispersion at the collision point to be $\eta_{x 0}=-0.0036 \mathrm{~m}$ and $\eta_{y 0}=-0.0011 \mathrm{~m}$ after adjusting the $Q D$. Eventually, taking into account the precision of determining $I_{x}$ and $I_{y}$, about $0.2 \mathrm{~A}$, and the dispersion, $\eta_{x 0}$ and $\eta_{y 0}$, we estimated the beam sizes $\left(\sigma_{e_{x 0}}\right.$ and $\left.\sigma_{e_{y 0}}\right)$ to be $7.6 \pm 0.5 \mu \mathrm{m}$ and $5.4 \pm 0.6 \mu \mathrm{m}$, as demonstrated in Fig. 10.

\section{B. Laser system}

As mentioned in Sec. I, we needed to develop a special $e^{-}$-laser colliding system with a short focal length for the JLC polarized positron system. In order to meet this requirement, we constructed a Compton chamber in which a pair of off-axis parabolic mirrors with a focal length of $150 \mathrm{~mm}$ are installed in the $e^{-}$-beam line $[11,12]$. Figure 11 shows a cross-sectional view of the Compton chamber, and Fig. 12 is a picture of the parabolic mirror. At both sides of the chamber, there are two beam-position monitors (BPM) to measure the positions of the $e^{-}$beam. As can be seen in Fig. 13, the $x$ axis stands for the axis of the parabola, the surface in the $y$-s plane is a circle, and the focal point is located at the origin. Each mirror has a through hole of $5 \mathrm{~mm}$ diameter at the center to pass the $e^{-}$beam and backscattered $\gamma$ rays. At the collision point, screen monitors can be inserted when the positions of the $e^{-}$and laser beams are measured. Near to the exit of the laser device, the laser beam is expanded two and a half times by an expander system consisting of concave and convex lenses. The thusobtained parallel beam with an rms size of $3.9 \mathrm{~mm}$ is guided into the chamber by three flat mirrors. In the chamber, the laser beam is focused by a parabolic mirror and makes a head-on collision with the $e^{-}$beam at the 

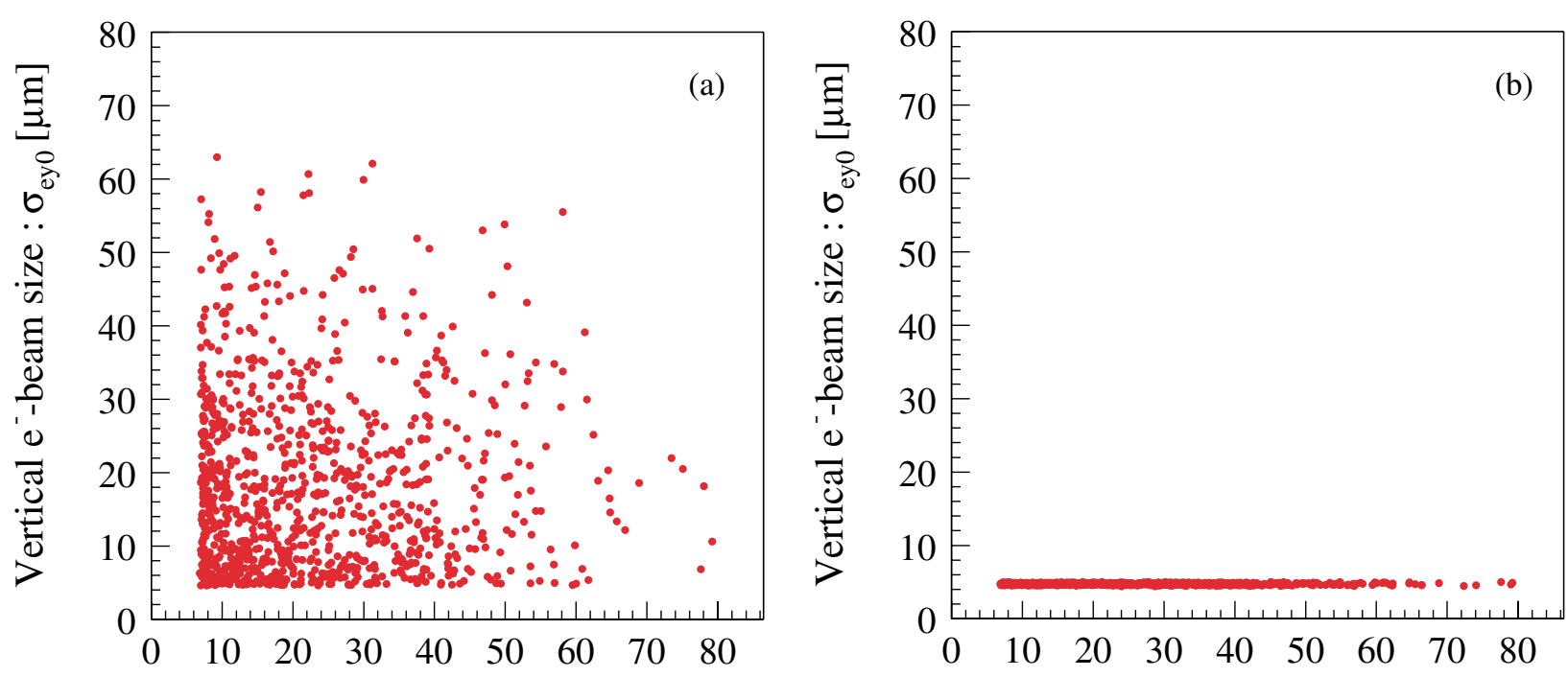

Horizontal $\mathrm{e}^{-}$-beam size $: \sigma_{\mathrm{ex} 0}[\mu \mathrm{m}]$
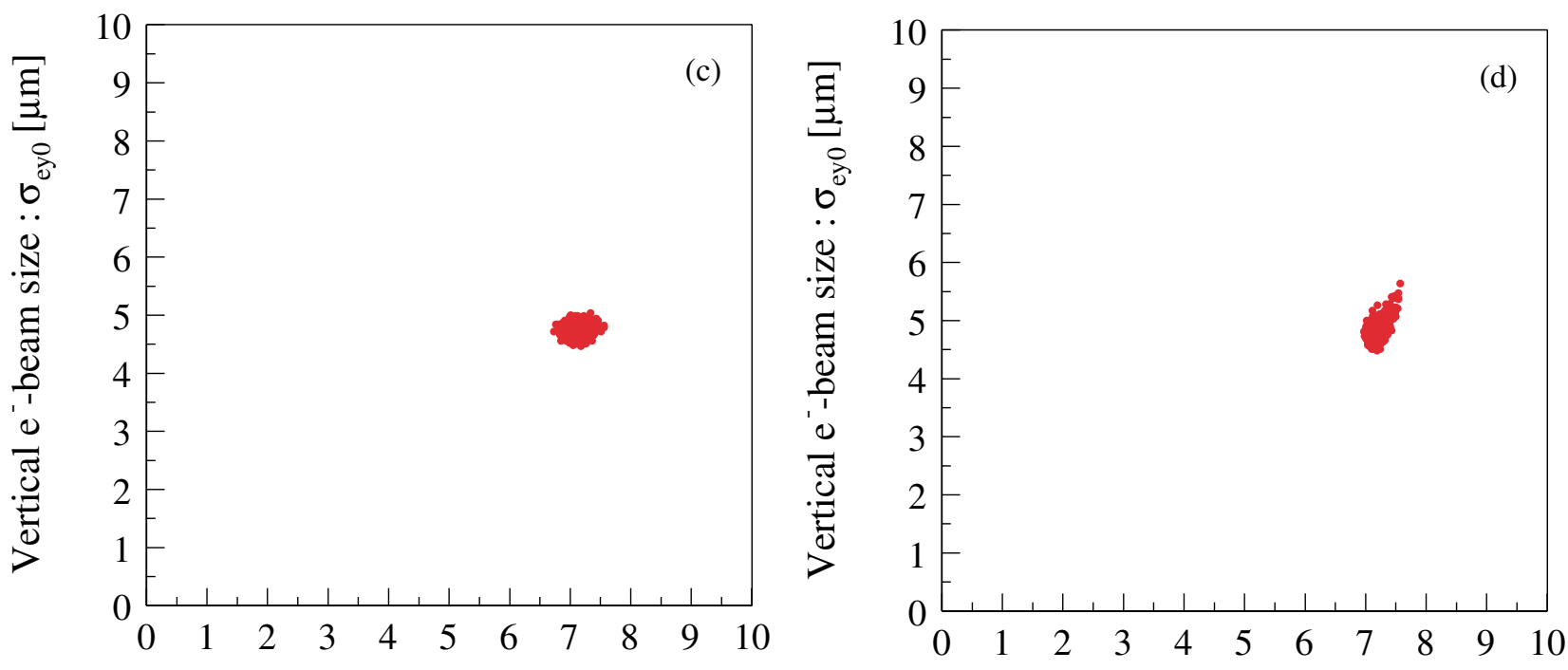

Horizontal $\mathrm{e}^{-}$-beam size $: \sigma_{\mathrm{ex} 0}[\mu \mathrm{m}]$

Horizontal e-beam size $: \sigma_{\mathrm{ex} 0}[\mu \mathrm{m}]$

FIG. 8. (Color) Two dimensional plot of vertical and horizontal beam sizes simulated by SAD: (a) without the error correction for the $Q$ magnets; (b) after the error correction for $Q Y$; (c) after the error correction for $Q Y$ and $Q X$; (d) after the error and dispersion corrections for $Q Y, Q X$, and $Q D$.

collision point. Since the parabolic mirror has a through hole, $18 \%$ of the laser beam is not reflected. The parameters of the laser are listed in Table I

Using an emittance $\epsilon_{\ell}$ of the laser beam and focal depth $\beta_{\ell}$, the laser-beam size at position $s$ is represented by

$$
\sigma_{\ell}(s)=\sqrt{\epsilon_{\ell}\left\{\beta_{\ell}+\frac{\left(s-s_{0}\right)^{2}}{\beta_{\ell}}\right\}} .
$$

In the case of an ideal laser beam, for which the spatial distribution can be represented as a Gaussian function, $\epsilon_{\ell}$ is given by

$$
\epsilon_{\ell}=\frac{\lambda}{4 \pi}
$$

where $\lambda$ is the wavelength. However, in the actual case, the deviation from the diffraction limit should be taken into account by introducing a factor $M^{2}$, such that

$$
\epsilon_{\ell}=M^{2} \frac{\lambda}{4 \pi}
$$

The spot size $\left(\sigma_{\ell_{0}}\right)$ at $s_{0}$ in the Compton chamber can be estimated using the relation $\sigma_{\ell_{0}}=\epsilon_{\ell} f / \sigma_{m}$, where $f$ is a focal length and $\sigma_{m}$ is an rms laser-beam size at the 

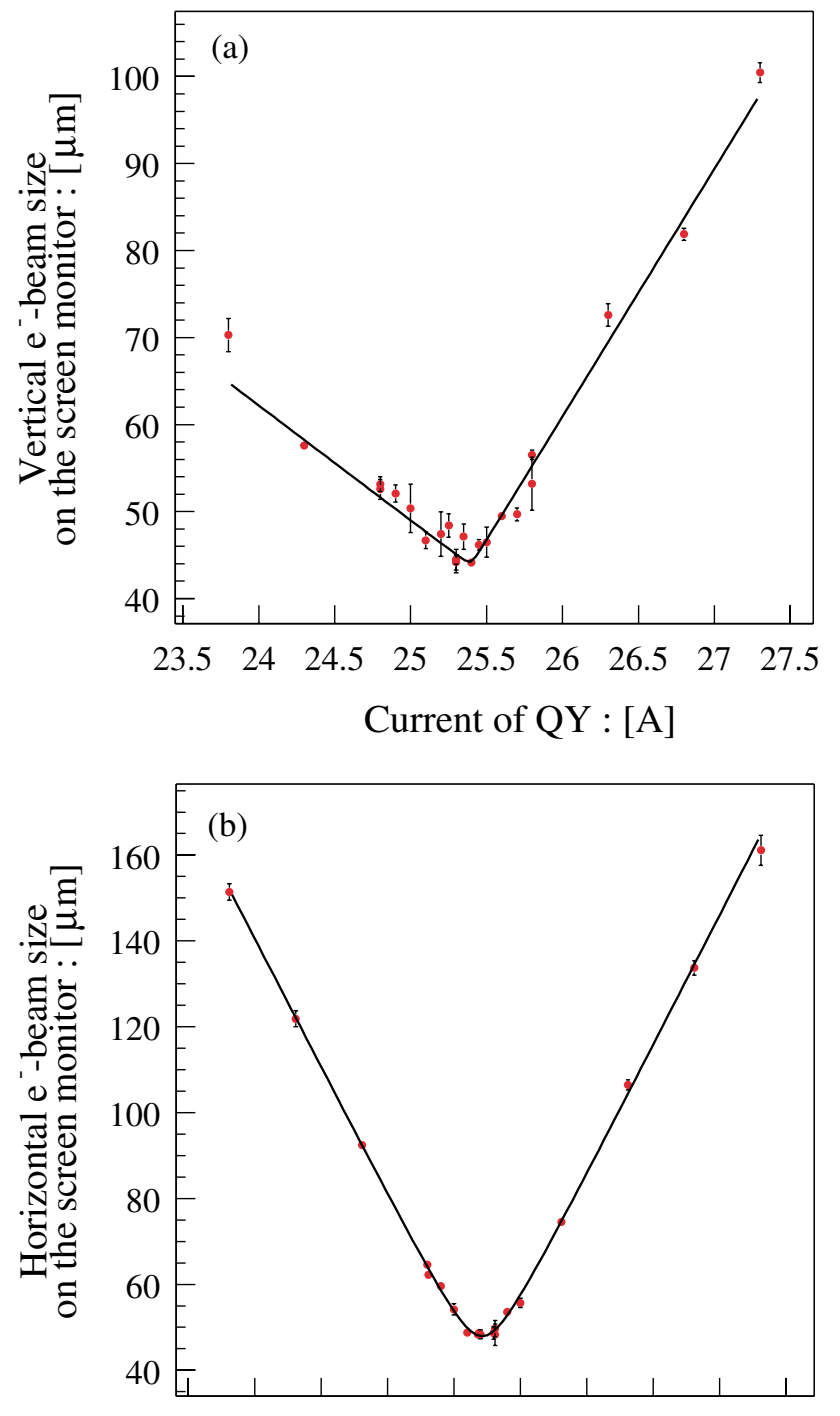

$\begin{array}{llllllllll}69 & 69.5 & 70 & 70.5 & 71 & 71.5 & 72 & 72.5 & 73 & 73.5\end{array}$

Current of QX : [A]

FIG. 9. (Color) Experimental results of the error corrections: Changing the current of the $Q$ magnets, the beam size on the screen monitors are measured at the collision point: (a) for the $y$ direction as a function of the current of $Q Y$; (b) for the $x$ direction as a function of the current of $Q X$.

parabolic mirror. However, when the focal length is $150 \mathrm{~mm}$, the spot size of the laser beam is about $6 \mu \mathrm{m}$, which is too small to directly measure using the chargecoupled device (CCD). Thus, in order to determine the laser emittance, we use a lens with a focal length of $2 \mathrm{~m}$ that allows us to produce the $100 \mu \mathrm{m}$ focal spot size. A measurement of the laser-beam size as a function of $s$ was carried out by varying the position of the CCD with a pixel size about $10 \mu \mathrm{m}$ around the focal point. The results are shown in Fig. 14 for both the horizontal and vertical directions. Since $\beta_{\ell} \simeq \epsilon_{\ell}\left(f / \sigma_{\mathrm{m}}\right)^{2}, \sigma_{\ell}(s)$ can be represented by

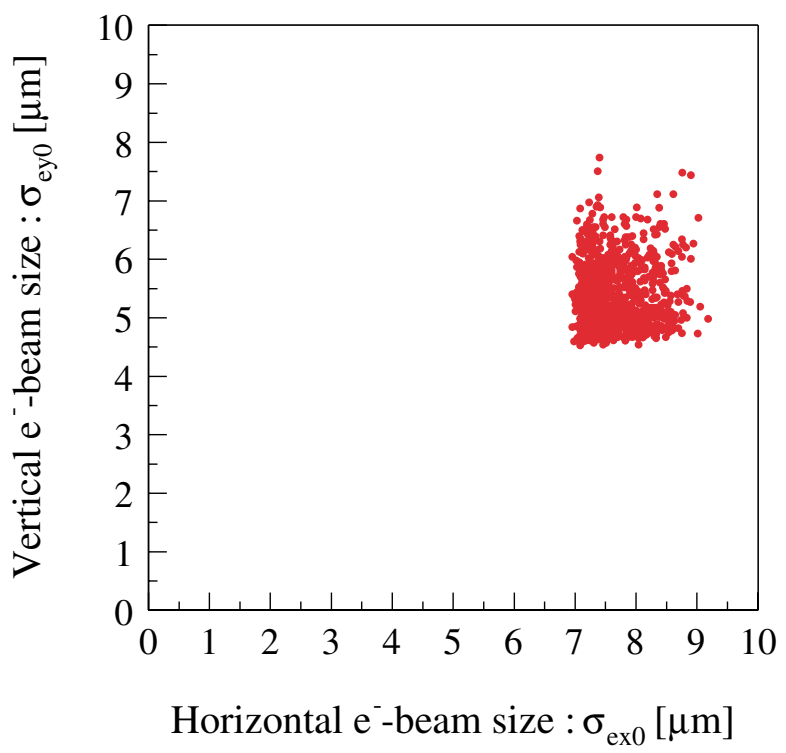

FIG. 10. (Color) Two dimensional plot of vertical and horizontal beam sizes simulated by SAD taking into account the precision of determining the optimum current of $Q Y, Q X$, and $Q D$.

$$
\sigma_{\ell}(s)=\sqrt{M^{2} \frac{\lambda}{4 \pi}\left\{M^{2} \frac{\lambda}{4 \pi}\left(\frac{f}{\sigma_{m}}\right)^{2}+\frac{4 \pi \sigma_{m}^{2}\left(s-s_{0}\right)^{2}}{M^{2} \lambda f^{2}}\right\}} .
$$

This function was fitted to the data to determine the free parameters, $M^{2}$ and $s_{0}$. We thus obtained $M_{x}^{2}=3.6$ and $M_{y}^{2}=3.3$, which permitted us to determine $\epsilon_{\ell_{x}}=(1.6 \pm$ $0.02) \times 10^{-7} \mathrm{rad} \mathrm{m}$ and $\epsilon_{\ell_{\mathrm{v}}}=(1.4 \pm 0.02) \times 10^{-7} \mathrm{radm}$. The spot size of the laser beam focused by the parabolic mirror was calculated using these parameters to be $\sigma_{\ell_{x 0}}=6.0 \pm 0.2 \mu \mathrm{m}$ and $\sigma_{\ell_{\mathrm{y} 0}}=5.3 \pm 0.2 \mu \mathrm{m}$.

The time distributions of the laser beam were measured using an oscilloscope with a sampling rate of $10 \mathrm{GHz}$, corresponding to a time resolution of $100 \mathrm{ps}$ and a streak camera with a time resolution of $0.2 \mathrm{ps}$ : The results are shown in Figs. 15(a) and 15(b). By fitting the Gaussian function to the distribution of Fig. 15(a), we obtained $\tau_{\ell}=3.6 \mathrm{~ns}$ in rms. However, as can be seen in Fig. 15(b), we still observe a microtime structure with a time interval of about 100 ps between two adjacent peaks caused by multimode oscillation. Since the laser pulse has a time jitter of about $500 \mathrm{ps}$, the change in the effective intensity of the laser beam induces fluctuations in the number of $\gamma$ rays, bunch by bunch.

\section{Results}

Because the time duration of the $\gamma$ rays, being equivalent to the bunch length of the $e^{-}$beam, is considerably short, i.e., 26 ps in rms, we cannot identify each $\gamma$ ray, and thus only the total number of $\gamma$ rays in each bunch can be measured. For $\gamma$-ray measurements, we provided a silica-aerogel Cherenkov counter with a refraction index 


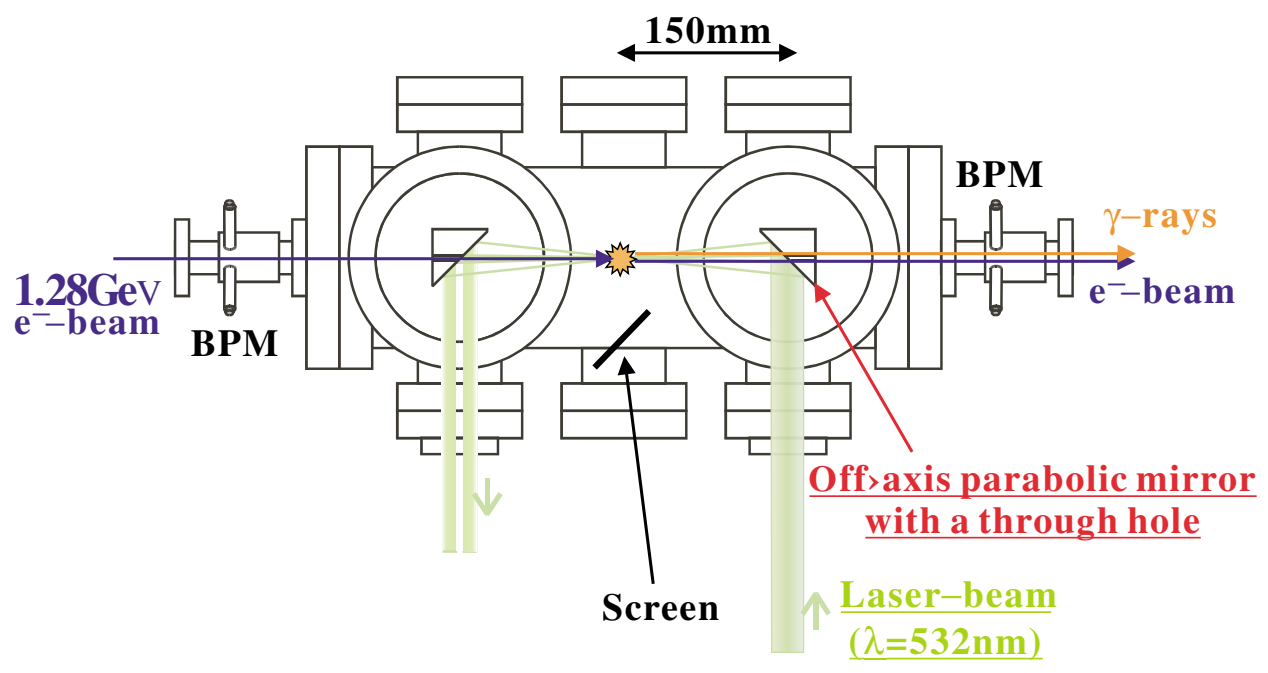

FIG. 11. (Color) Top cross-sectional view of the Compton chamber.

of $n=1.015$, being sensitive to those electrons (positrons) with the kinetic energy higher than $2.47 \mathrm{MeV}$. The cross section of the silica aerogel is $100 \times 100 \mathrm{~mm}^{2}$ and the thickness is $50 \mathrm{~mm}$. At the front of the detector, an aluminum plate of $3 \mathrm{~mm}$ thickness is placed to convert $\gamma$ rays into $e^{-}-e^{+}$pairs. The conversion efficiency and geometrical acceptance of the counter is estimated using the detector simulation code GEANT [16]. The calibration of the counter for a single minimum ionizing particle (MIP) was done by using cosmic rays. In the $\gamma$-ray detection, the applied high voltage (HV) to a photomul-

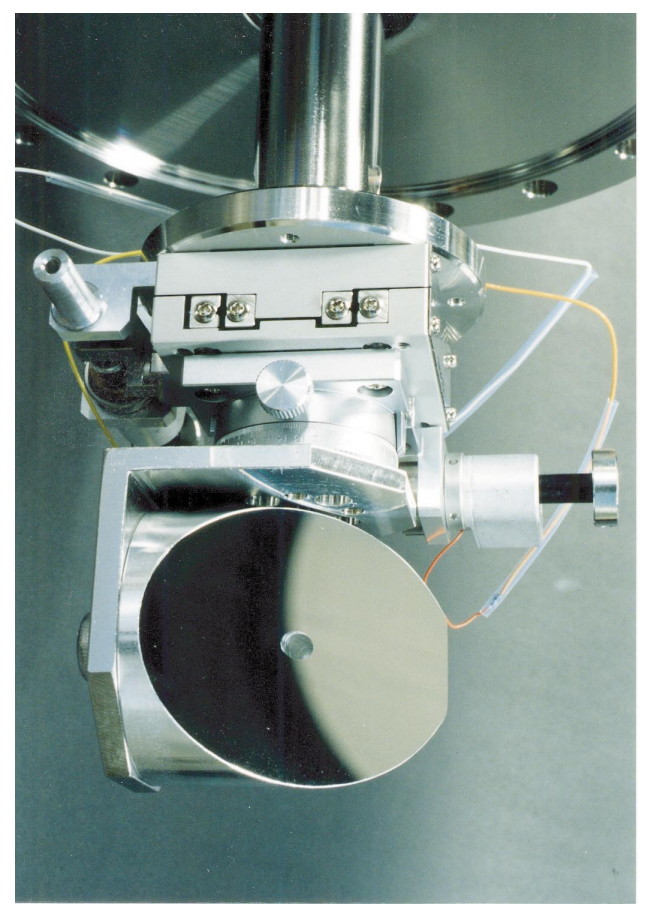

FIG. 12. (Color) Picture of the parabolic mirror with a through hole at the center. tiplier tube of the counter was set at a much lower voltage than that applied in the single MIP calibration, in order to detect large numbers of electrons and positrons created by the $\gamma$ ray. To correct the gain difference caused by deference of the applied HV, a HV-gain curve was measured using a light-emitting diode as a standard light source.

Table I puts together the measured and designed parameters of the $e^{-}$and laser beams. The position jitters $\Delta_{e_{i}}\left(\Delta_{\ell_{i}}\right)$ of the $e^{-}$beam (laser beam) with $i=x, y$ are observed using screen monitors inserted at the collision point. The angle jitters of the $e^{-}$beam in $\operatorname{rms}\left(\theta_{x}\right.$ and $\left.\theta_{y}\right)$ are measured by the BPMs at both sides of the Compton chamber. In order to accomplish a high collision luminosity, we must create a small spot size of both $e^{-}$and laser beams and achieve a large overlap of the temporal and spatial distributions between the $e^{-}$and laser beams as well. Hence, we attempted to reduce the temporal offsets by maximizing the production rate of $\gamma$ rays by scanning the timing of the laser $Q$ switch and the spatial offsets by adjusting the angle of the parabolic mirror. Figure 16 shows the detected number of $\gamma$ rays as a function of the $x$ and $y$ distances between the $e^{-}$and laser beams. The distances are measured by a screen located at the collision point which is the focal point of both the $e^{-}$and the laser beams. We fitted doubleGaussian functions to each distribution and obtained the $\sigma$ value (rms) of the narrower Gaussian distribution, i.e., $\Gamma_{x}=44 \mu \mathrm{m}$ and $\Gamma_{y}=25 \mu \mathrm{m}$. It is discussed in Sec. III that the peak in Fig. 16(a) deviates from zero. The number of photons corresponding to the signals and backgrounds was measured when the laser beam was switched on and off, respectively. Figure 17 demonstrates that the signal was clearly separated from the background. The mean value in the number of the Compton scattered $\gamma$ rays was $N_{\gamma_{\exp }}=(2.0 \pm 0.03 \pm 0.3) \times 10^{5} \gamma \mathrm{s} /$ bunch, with 0.03 and 0.3 being the statistical and systematic errors. It is noted that the signal distribution has a wide width (discussed in 


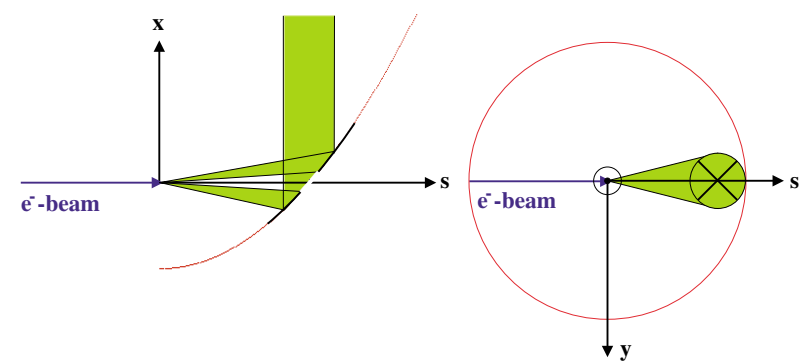

FIG. 13. (Color) Shape of the parabolic mirror and the laser focusing.

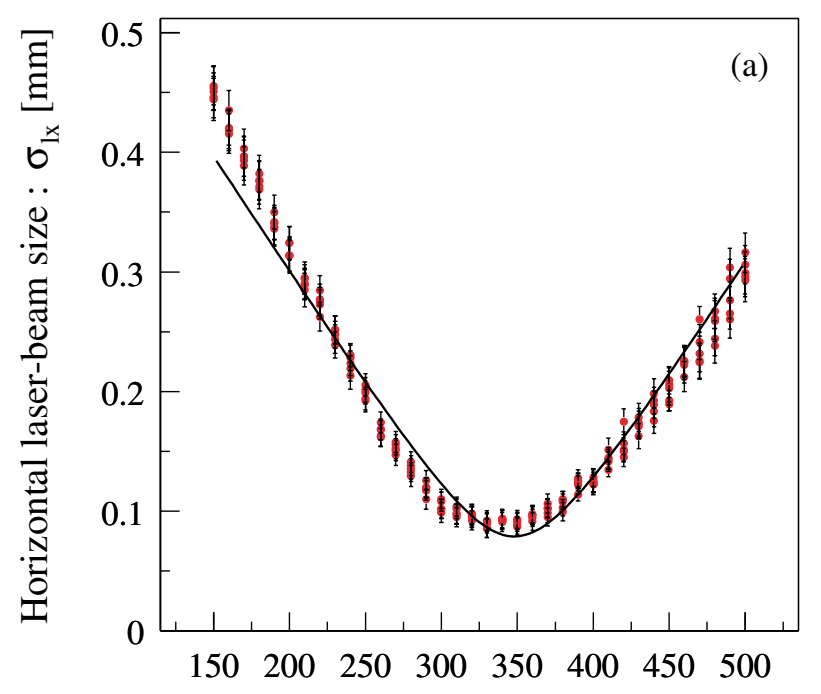

Relative distance from a lens : $\mathrm{s}$ [mm]

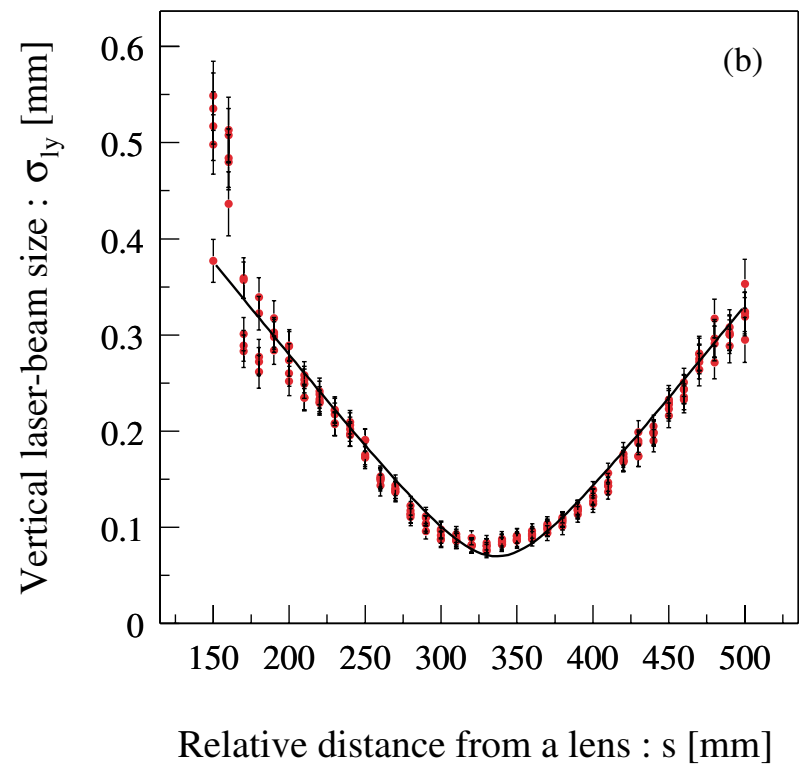

FIG. 14. (Color) Laser-beam size as a function of the relative distance from a lens of $f=2000 \mathrm{~mm}$ for the laser emittance measurement: (a) for the horizontal direction; (b) for the vertical direction.
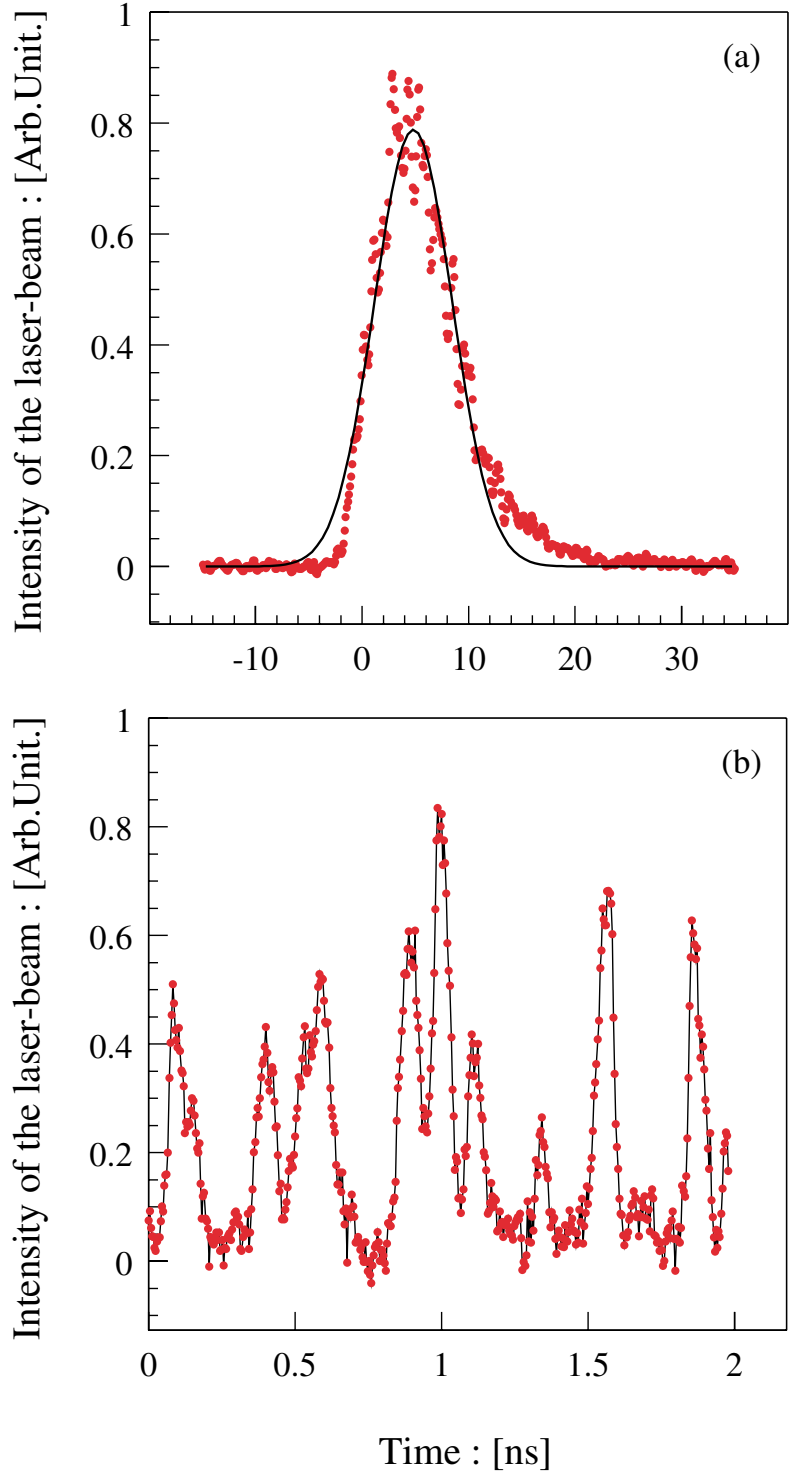

FIG. 15. (Color) Time distribution of the laser beam measured: (a) over the entire extent of the laser pulse using an oscilloscope; $\tau_{\ell}=3.6 \mathrm{~ns}$ in rms; (b) at the central region of the laser pulse by a streak camera.

Sec. IV in terms of the time structures of the laser pulse). In order to make a cross-check of the number of $\gamma$ rays, a $\mathrm{Si}$ detector is also utilized. Here the calibration was made from energy necessary to make the electron-hole pair $(3.6 \mathrm{eV})$ and the gain of the amplifier. Consequently we confirmed that the number measured by the Si detector were in good agreement with that measured by the Cherenkov counter within the error.

The quality of a radiation source is evaluated by the brightness $(B)$ defined as

$$
B=\frac{N_{\gamma}}{(2 \pi)^{5 / 2} \delta_{x} \delta_{y} \theta_{\gamma_{x}} \theta_{\gamma_{y}} \tau_{e}} /\left(\operatorname{mrad}^{2} \mathrm{~mm}^{2} 0.1 \% \text { bandwidth } \mathrm{s}\right)
$$




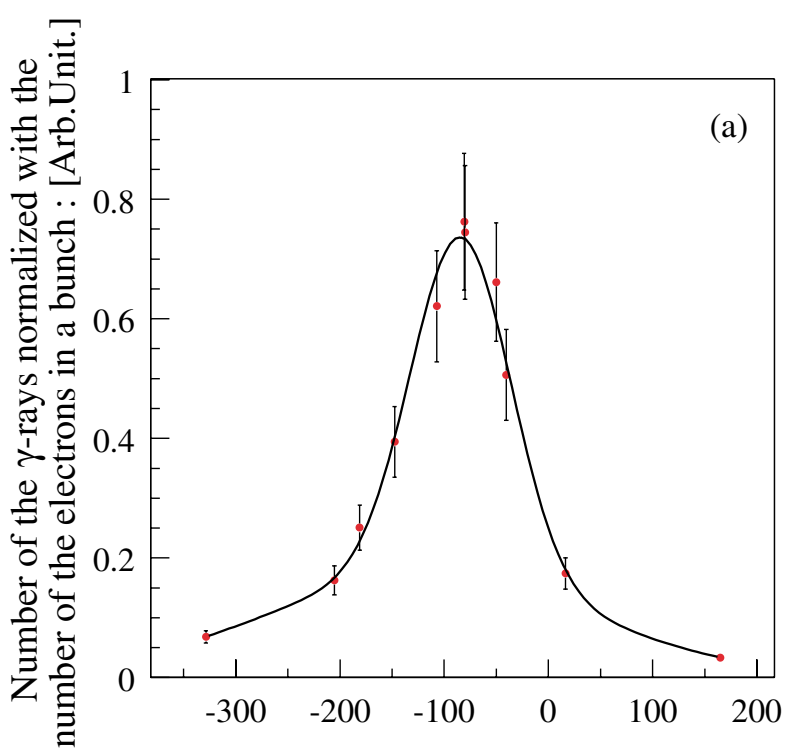

Horizontal distance between the positions of the $\mathrm{e}^{-}-$and laser-beams : $[\mu \mathrm{m}]$

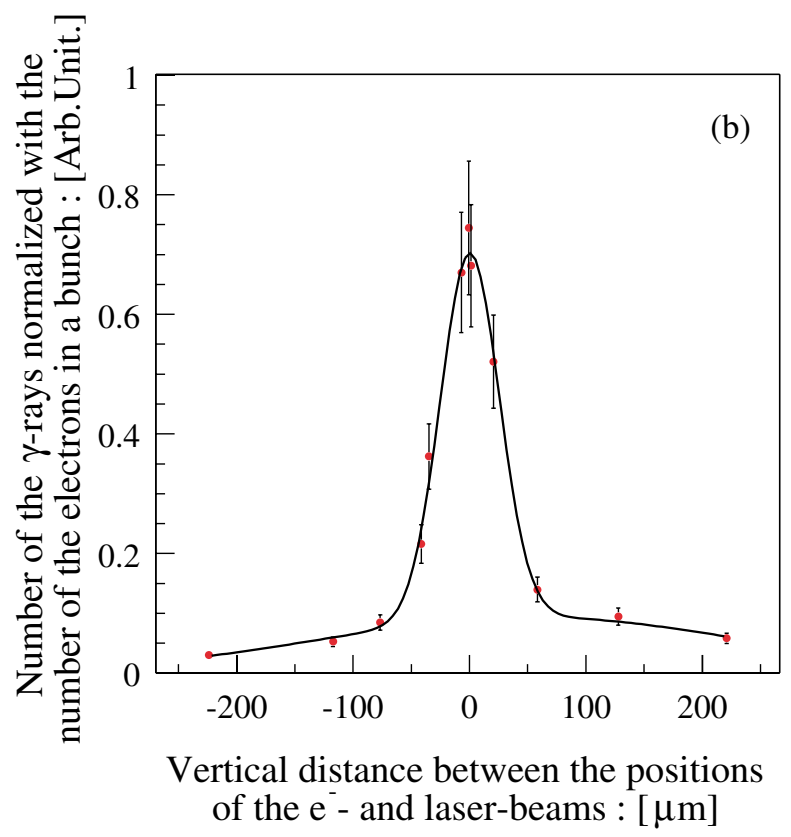

FIG. 16. (Color) Result of the position scan: (a) for the horizontal direction, $\Gamma_{x}=44 \mu \mathrm{m}$; (b) for the vertical direction, $\Gamma_{y}=25 \mu \mathrm{m}$.

where $\delta_{i}(i=x, y)$ is the overlap size between the $e^{-}$and laser beams, $\theta_{\gamma_{i}}(i=x, y)$ is the divergence of $\gamma$ rays, and $\tau_{e}$ is the bunch length of the $e^{-}$beam. Assuming Gaussian distributions for the sizes and jitters of the $e^{-}$ and laser beams, we derive $\delta_{i}$ as

$$
\delta_{i}=\sqrt{\frac{\left(\overline{\left.\sigma_{e_{i 0}}^{2}+\Delta_{e_{i}}^{2}\right)\left(\sigma_{\ell_{i 0}}^{2}+\Delta_{\ell_{i}}^{2}\right)}\right.}{\sigma_{e_{i 0}}^{2}+\Delta_{e_{i}}^{2}+\sigma_{\ell_{i 0}}^{2}+\Delta_{\ell_{i}}^{2}}} \quad(i=x, y) .
$$

Because the $e^{-}$-beam divergences are negligible com-

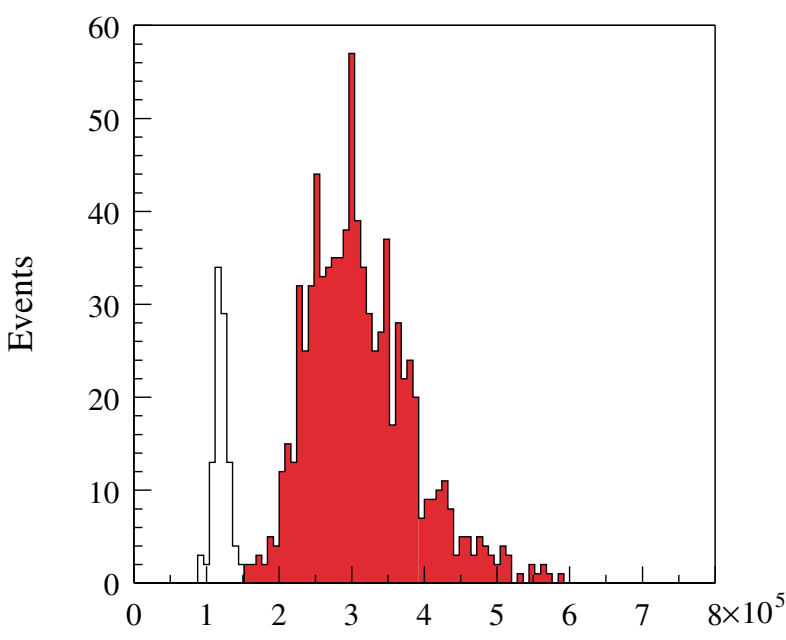

Number of the $\gamma$-rays : $\mathrm{N}_{\gamma}[\gamma \mathrm{s} /$ bunch $]$

FIG. 17. (Color) Number of detected $\gamma$ rays; the white histogram shows the laser-off signal and the red one shows the laseron signal.

pared with the angle jitter, it may be assumed that $\theta_{\gamma_{i}}$ is equal to the angle jitter of the $e^{-}$beam $\left(\theta_{e_{i}}\right)$, and the bandwidth $0.1 \%$ has an energy spread of $0.056 \mathrm{MeV}$ at the highest energy edge $(56 \mathrm{MeV})$. Using the numerical values given in Table I, we calculated the brightness as $1.7 \times$ $10^{18} /\left(\mathrm{mrad}^{2} \mathrm{~mm}^{2} 0.1 \%\right.$ bandwidth s $)$ near $56 \mathrm{MeV}$.

\section{NUMERICAL MODEL}

The expected number of $\gamma$ rays is calculated by the product of the collision luminosity and the total cross section of the Compton scattering, i.e., $637 \mathrm{mb}$. The luminosity $(\mathcal{L})$ at each bunch is represented by

$$
\mathcal{L}=2 \iiint \int_{-\infty}^{\infty} \rho_{e}(x, y, s, t) \rho_{\ell}(x, y, s, t) d x d y d s d c t,
$$

where, $\rho_{e}$ and $\rho_{\ell}$ are the density functions of the $e^{-}$and laser beams, respectively. When the time distributions are described by the Gaussian distributions, $\rho_{e}$ and $\rho_{\ell}$ are given by

$$
\begin{aligned}
& \rho_{e}(x, y, s, t)=\frac{N_{e} \rho_{e}^{\prime}(x, y, s)}{\sqrt{2 \pi} \tau_{e}} \exp \left\{-\frac{(s-c t)^{2}}{2 \tau_{e}^{2}}\right\}, \\
& \rho_{\ell}(x, y, s, t)=\frac{N_{\ell} \rho_{\ell}^{\prime}(x, y, s)}{\sqrt{2 \pi} \tau_{\ell}} \exp \left\{-\frac{(s+c t)^{2}}{2 \tau_{\ell}^{2}}\right\},
\end{aligned}
$$

where $N_{i}(i=e, \ell)$ are a numbers of electrons and photons contained in one bunch, $\rho_{i}^{\prime}(i=e, \ell)$ is the spatialdensity function, and $\tau_{e}\left(\tau_{\ell}\right)$ is the bunch length of the $e^{-}$ (laser) beam. Because $\tau_{e}(=26 \mathrm{ps}) \ll \tau_{\ell}(=3.6 \mathrm{~ns})$, the temporal density function of the $e^{-}$beam is approximated by 


$$
\frac{1}{\sqrt{2 \pi} \tau_{e}} \exp \left\{-\frac{(s-c t)^{2}}{2 \tau_{e}^{2}}\right\} \approx \delta(s-c t)
$$

and thus

$$
\int_{-\infty}^{\infty} \rho_{e}(x, y, s, t) \rho_{\ell}(x, y, s, t) d c t=\rho_{e}^{\prime} \rho_{\ell}^{\prime} \frac{N_{e} N_{\ell}}{\sqrt{2 \pi} \tau_{\ell}}\left\{-\frac{(2 s)^{2}}{2 \tau_{\ell}^{2}}\right\} .
$$

The spatial-density function of the $e^{-}$beam $\left(\rho_{e}^{\prime}\right)$ is given by

$$
\begin{aligned}
\rho_{e}^{\prime}(x, y, s)= & \frac{1}{\sqrt{2 \pi} \sigma_{e_{x}}(s)} \exp \left\{-\frac{x^{2}}{2 \sigma_{e_{x}}^{2}(s)}\right\} \frac{1}{\sqrt{2 \pi} \sigma_{e_{y}}(s)} \\
& \times \exp \left\{-\frac{y^{2}}{2 \sigma_{e_{y}}^{2}(s)}\right\},
\end{aligned}
$$

where the $e^{-}$-beam size $\left(\sigma_{e_{x, y}}\right)$ is represented by Eq. (1). Because the laser beam is focused by a parabolic mirror with a through hole, we must consider the laser-beam distribution, as shown in Fig. 18(b):

$$
\rho_{\ell}^{\prime}\left(r, r_{\ell}, \sigma_{\ell}, s\right)=A(s) \exp \left\{-\frac{r^{2}}{2 \sigma_{\ell}^{2}}\right\} a(r),
$$

where $A(s)$ is a normalization factor, $\sigma_{\ell}(s)$ is the laserbeam size, and $r_{\ell}(s)$ is the hole radius of the laser beam at $s$. The factor $a(r)$ stands for a step function, defined as

(a)

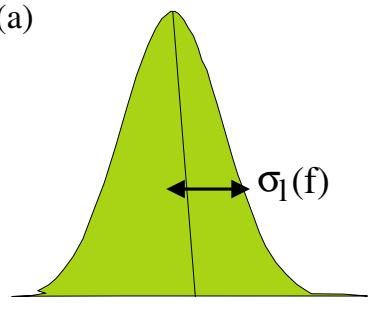

(c)

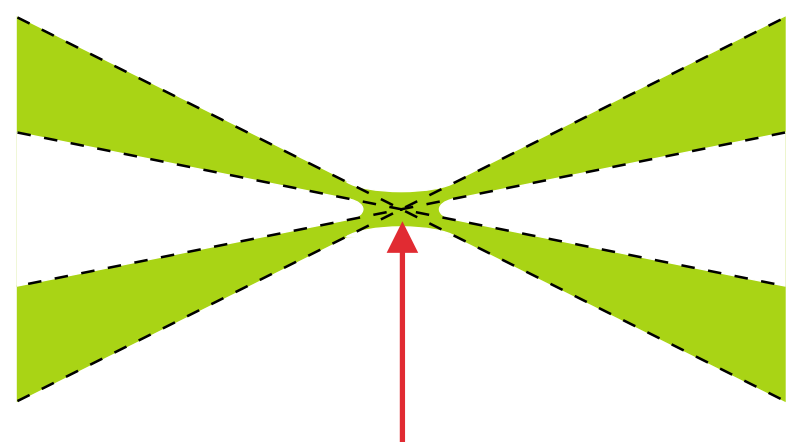

\section{Collision point}

FIG. 18. (Color) Distribution of the laser beam: (a) before injection on the parabolic mirror; (b) after injection on the parabolic mirror; (c) around the collision point.

$$
a(r)= \begin{cases}1 & {\left[r \geq r_{\ell}(s)\right]} \\ 0 & {\left[r<r_{\ell}(s)\right] .}\end{cases}
$$

Using the focal length $(f), \sigma_{\ell}(s)$ and $r_{\ell}(s)$ are described as

$$
\sigma_{\ell}(s)=\frac{\sigma_{\ell}(f) s}{f}, \quad r_{\ell}(s)=\frac{r_{\ell}(f) s}{f},
$$

where $\sigma_{\ell}(f)=3.9 \mathrm{~mm}$ is the rms size of the laser beam and $r_{\ell}(f)=2.5 \mathrm{~mm}$ is the through hole size, both at the parabolic mirror. In order to make a complete evaluation of a laser-beam profile near the focal point, it is necessary to perform Kirchhoff integration with partial coherence of the laser beam used in the experiment. However, for the laser wavelength of $532 \mathrm{~nm}$ and the geometry of the mirror, the calculation based on the simulation code GLAD [17] for analyzing optics clarifies that the existence of interference fringes caused by diffraction can be negligible. Using a shape convoluted with a distribution described in geometrical optics and the spot size $\left(\sigma_{\ell_{0}}\right)$ calculated by Eq. (2), the spatial distribution is approximated as

$\rho_{\ell}^{\prime}(x, y, s)=\iint_{-\infty}^{\infty} \frac{\rho_{\ell}^{\prime}\left(r, r_{\ell}, \sigma_{\ell}, s\right)}{2 \pi \sigma_{\ell_{0}}^{2}} \exp \left(-\frac{x^{\prime^{2}}+y^{\prime^{2}}}{2 \sigma_{\ell_{0}}^{2}}\right) d x^{\prime} d y^{\prime}$,

where $r=\sqrt{\left(x-x^{\prime}\right)^{2}+\left(y-y^{\prime}\right)^{2}}$. Here $\sigma_{l_{0}}$ is the average value of $\sigma_{l_{x_{0}}}(=6.0 \mu \mathrm{m})$ and $\sigma_{l_{y_{0}}}(=5.3 \mu \mathrm{m})$. To substitute Eqs. (6) and (7) into Eq. (5), we described the luminosity with the known parameters of the $e^{-}$and laser beams, given in Table I, and obtained the number of $\gamma$ rays, $5.1 \times 10^{5} \mathrm{\gamma s} /$ bunch, which is 2.5 times larger than the experimental value. As shown in Fig. 16, the profile of the position scan in the horizontal direction is wider than that in the vertical direction and in addition the peak in the horizontal scan is shifted from the zero. It may be understood in relation with the shape of the parabolic mirror surface, in which the shape in the $x-s$ plane is parabolic, while that in the $y$-s plane is circular. If the $e^{-}$orbit deviates from the central orbit in the $x-s$ plane, or the laser beam is injected on the parabolic mirror not in parallel with the mirror axis, i.e., the $x$ axis, we are compelled to readjust the laser beam in order to accomplish the maximum overlap between the $e^{-}$and laser beams. In this case, because the parabolic mirror is rotated around the $y$ axis, the $x$ focal point also shifts along the $s$ direction. On the other hand, when the mirror is rotated around the $x$ axis, a shift of the focal point along the $s$ direction does not take place. Consequently, the $x$ focal point deviates from the collision point, resulting in a deformation of the normal shape of the laser spot. Using a simplified version of a numerical model in which the through hole of the mirror is neglected, we can estimate the intensity variation in terms of the angle 
between the injected laser beam and the mirror axis when the parabolic mirror is rotated around the $y$ axis. It is then found that to obtain the reduction of $\gamma$-ray intensity which agrees with that observed in the experiment, we may introduce an angular deviation of $8 \mathrm{mrad}$ in the $x-s$ plane, which can shift the focal point in the $x$-s plane from that in the $y-s$ plane by $2.4 \mathrm{~mm}$. Note that if laser profile is unchanged, the luminosity reduction caused by the $8 \mathrm{mrad}$ deviation of the crossing angle is negligible. The numerical model clarified that the reduction was caused by the deformation of a laser spot. Such an amount of the angular deviation may occur if we consider the current optical system, which is not equipped with sufficiently accurate control devices to adjust the axis of the incident laser beam, which should be parallel to the axis of the parabolic mirror. This model allows us also to predict the dependence of the $\gamma$-ray yield upon the spatial overlap between the laser and the electron beams. The simulated plot in Fig. 19(b) is in good agreement with the measured vertical distribution shown in Fig. 16(b). A possible $8 \mathrm{mrad}$ angular misalignment of the beams in a horizontal plane explains a relatively broad experimental distribution with $\Gamma_{x}=44 \mu \mathrm{m}$ shown in Fig. 16(a) to compare with the simulated $\Gamma_{x}^{M}=29 \mu \mathrm{m}$ [see Fig. 19(a)]. It is remarked that the shift of the center emerging only in the horizontal distribution, as shown in Fig. 16, is also reproduced by this model, in which $8 \mathrm{mrad}$ is taken into account. From the above consideration, we estimate that deformation of the laser spot, which is caused by deviation between the injected laser-beam axis and the mirror axis, causes the 2.5 times discrepancy of the number of $\gamma$ rays between calculation and data.

As mentioned in Sec. II B, we suppose that the wide fluctuation of the number of $\gamma$ rays shown in Fig. 17 is caused by a change in the effective intensity of the laser beam. In order to understand the relation between the wide fluctuation of the number of $\gamma$ rays and the microtime structures of the laser beam, we formulate the time-dependent laser intensity caused by multimode oscillation. The standing waves of the longitudinal modes in the laser resonator, $a_{i} \sin \left(\omega_{i} t+\phi_{i}\right)$, for each mode, where $\omega_{i}, \phi_{i}$, and $a_{i}$ are the angular frequency, random phase angle, and amplitude, give rise to the time distribution of the intensity,

$$
P_{\ell}^{\prime}(t)=\left\{\sum_{i=1}^{ \pm n} a_{i} \sin \left(\omega_{i} t+\phi_{i}\right)\right\}^{2} .
$$

In the laser resonator, the mode $\left(q_{i}\right)$ and the angular frequency $\left(\omega_{i}\right)$ are calculated as $q_{i}=q_{0}+i$, with $q_{0}=$ $2 L / \lambda$ and $\omega_{i}=q_{i} \pi c / L$, where $L$ stands for the length of the laser resonator and $c$ is the light velocity. Under a reasonable assumption that the intensity distribution of each mode follows a Gaussian function fluctuating by $\delta \nu=30 \mathrm{GHz}$ around $\nu_{0}$, the amplitude $\left(a_{i}\right)$ for each mode is given by

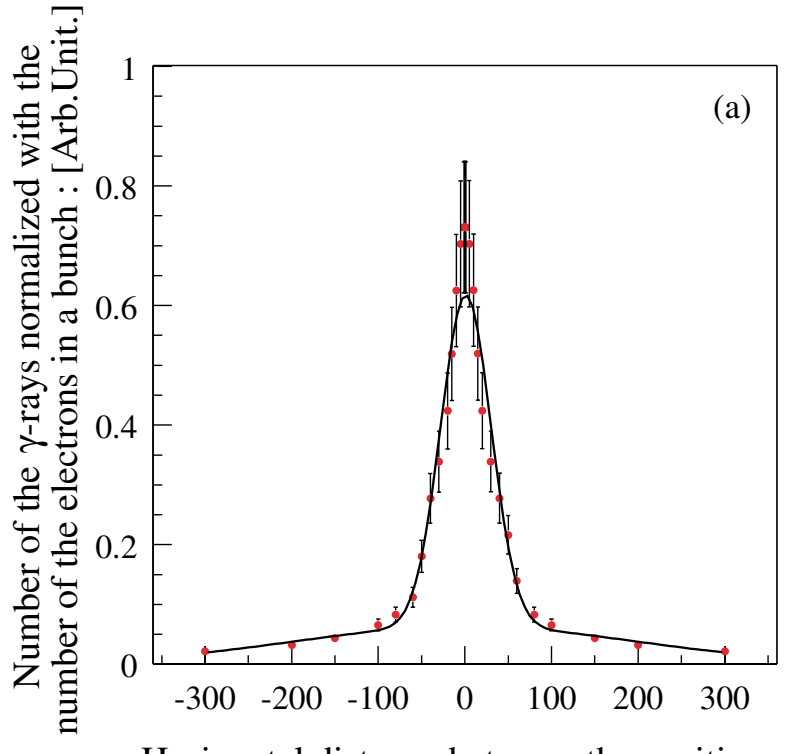

Horizontal distance between the positions of the $\mathrm{e}^{-}$- and laser-beams : $[\mu \mathrm{m}]$

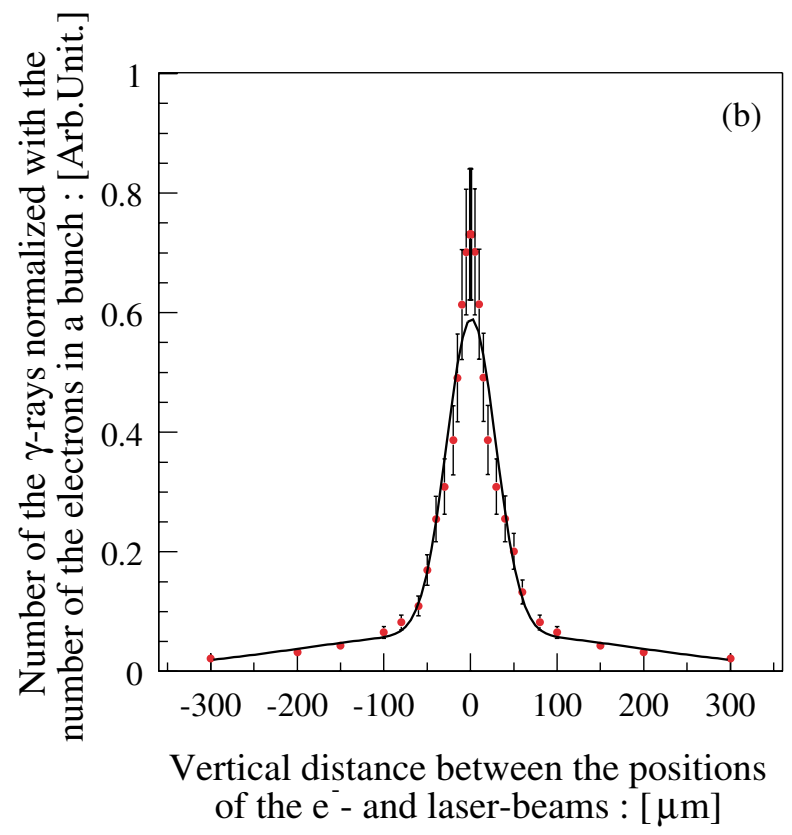

FIG. 19. (Color) Result of the position scan calculated by the numerical model: (a) for the horizontal direction, $\Gamma_{x}^{M}=$ $29 \mu \mathrm{m}$; (b) for the vertical direction, $\Gamma_{y}^{M}=28 \mu \mathrm{m}$.

$$
a_{i}=a \exp \left\{-\frac{\left(\nu_{i}-\nu_{0}\right)^{2}}{2 \sigma_{\nu}^{2}}\right\},
$$

where $\nu_{i}=\omega_{i} /(2 \pi), \sigma_{\nu}=\delta \nu / 2.35$, and $a$ is a normalized factor. Figure 20 shows the calculated time distribution of the laser intensity with parameters $L=0.52 \mathrm{~m}$, $\delta \nu=30 \mathrm{GHz}$ (FWHM), and $\lambda=1064 \mathrm{~nm}$. Thus, the effective intensity of the laser beam,

$$
P_{\ell}=P_{\ell}^{\prime}(t) \exp \left\{-\frac{(c t)^{2}}{2 \tau_{\ell}^{2}}\right\}
$$




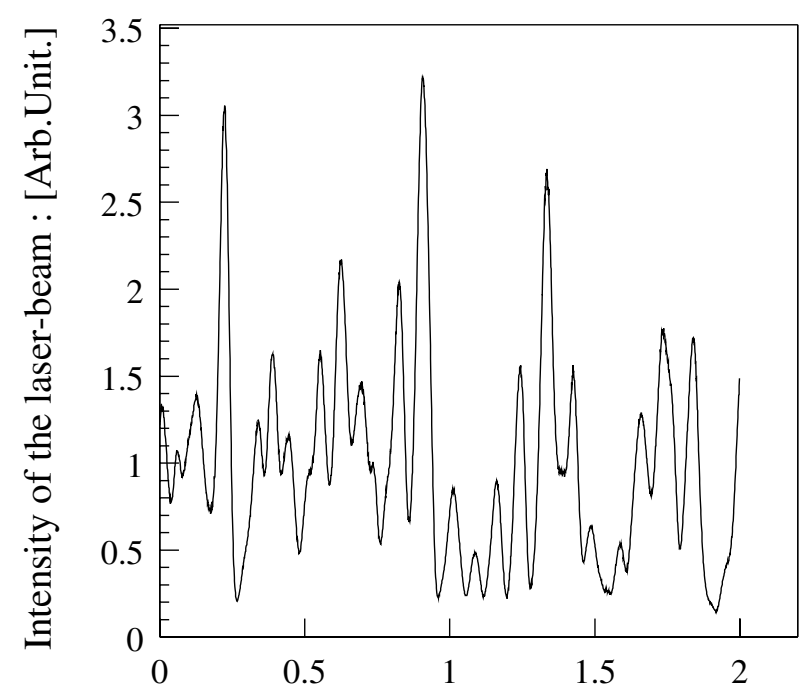

Time : [ns]

FIG. 20. Time distribution of the laser beam by the numerical model.

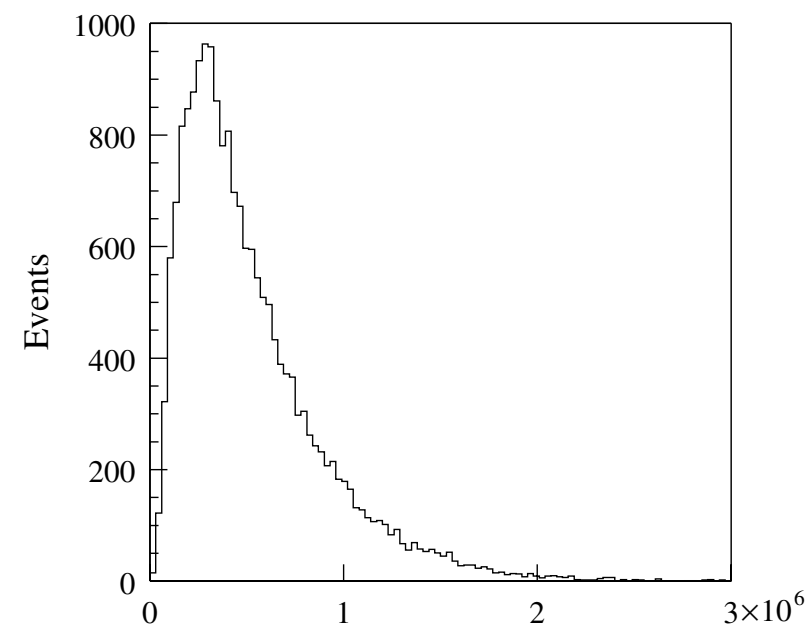

Number of the $\gamma$-rays : [ $\gamma$ s/bunch $]$

FIG. 21. Number of the generated $\gamma$ rays calculated by the numerical model.

gives the number of the $\gamma$ rays generated on a bunchby-bunch basis, as plotted in Fig. 21, where the broad $\gamma$-ray distribution observed in the experiment is also reproduced.

\section{CONCLUSIONS AND FUTURE PROSPECTS}

Based on the proposed method of generating highly polarized positrons [4], we have been pursuing a series of proof-of-principle experiments at both KEK and BNL. In accordance with the conceptual design for the polarized $e^{+}$beam of the JLC [10], we established a special colli- sion system with a short focal length for laser pulses. We constructed a Compton chamber which accommodates parabolic mirrors with a focal length of $150 \mathrm{~mm}$ to achieve a laser-beam size of $6 \mu \mathrm{m}[12,18]$. This laser focusing system necessarily requires an extremely small spot size of the $e^{-}$beams to accomplish efficient laserelectron collisions. By using SAD, into which the measured values of the emittances and momentum spreads for the $e^{-}$beam are input, we successfully found a special optics, the collision optics, to minimize the $e^{-}$-beam size to be different from the normal optics designed for emittance measurements at the collision point. To realize collision optics, we were able to find a practical method in which each $Q$ magnet permitted the $e^{-}$-beam size to be minimized independently in the horizontal and vertical directions. Actually, the $e^{-}$-beam sizes measured in this manner, i.e., $\sigma_{e_{x 0}}=7.6 \mu \mathrm{m}$ and $\sigma_{e_{y 0}}=5.4 \mu \mathrm{m}$, are considerably close to the designed values for the current optics of the damping ring. In comparison with the previous experiment [8], significant improvements in the luminosity were demonstrated, giving rise to an increase in the $\gamma$-ray yield by about 2 orders, i.e., a peak intensity of $8 \times 10^{15} / \mathrm{s}$, corresponding to a brightness of $1.7 \times$ $10^{18} /\left(\mathrm{mrad}^{2} \mathrm{~mm}^{2} 0.1 \%\right.$ bandwidth $\left.\mathrm{s}\right)$ around an energy of $56 \mathrm{MeV}$.

In order to precisely understand the collision process, we developed a numerical model in which realistic parameters are input for both the $e^{-}$and laser beams. This model brings plenty of important information, which will be helpful for further improvements of the luminosity as well as for designing the collision system at the JLC. The model elucidates that a slight deviation of the parallelism between the incident laser beam and the axis of the parabolic mirror takes place, and thus causes a reduction of the luminosity because of a deformation of the laserbeam shape around the collision point. Based on the thus revealed knowledge, we are now designing a new collision system which incorporates wire scanners, beamposition monitors, screen monitors, and knife-edge scanners to measure the beam positions and sizes for both $e^{-}$ and laser beams. This improved system will enable us to make accurate determinations of the beam axes and thus to maximize the luminosity.

We will extrapolate an expected $\gamma$-ray intensity for the JLC by taking into account the different collision scheme between the current system and the design one for the JLC, in which a multiple collision system is proposed $[9,10]$. Actually the designed system will gain the following factors: 10 for the electron-beam intensity, $10^{3}$ for the laser-beam intensity, 20 for the $\mathrm{CO}_{2}$ laser wavelength of $10 \mu \mathrm{m}$ which is 20 times as long as that of the current $\mathrm{Nd}$ :YAG laser, and 50 for the multiple collision system instead of the single collision system in the present experiment. Consequently we can attain an enhancement factor of $N_{\mathrm{en}}=10^{7}$, thereby resulting in the $\gamma$ intensity of $2 \times 10^{12} /$ bunch which is consistent with the designed 
value of $5 \times 10^{11} /$ bunch. Note that the obtained brightness brings further enhancement to the designed brightness by more than $N_{\text {en }}$, because the divergence and bunch width of the generated $\gamma$ ray in the current experiment is expected to be highly improved owing to the higher energy, smaller emittance, and shorter bunch width of the designed $e^{-}$beam.

As a next step of the polarized positron project, we will measure the spin polarization of $\gamma$ rays and positrons $[19,20]$. The polarization measurement can be performed based on the fact that the cross section of the Compton scattering of a $\gamma$ ray on a free electron in a magnetized iron is dependent of the relative spin direction between the $\gamma$ ray and the electron [21-23]. Owing to an extremely short bunch width of the $e^{-}$beam in our experiment, we cannot measure scattered $\gamma$ rays or recoil electrons on an event-by-event basis. Hence, we have been developing a method based on the fact that the total intensity of $\gamma$ rays transmitted through a magnetized iron also has spin dependence. However, the asymmetry of this method is relatively small, e.g., about $1 \%$, and thus further improvement of the luminosity of the collision should be needed. This can be accomplished by introducing a more intense, mode-locked laser and a new collision system of long focal length which is equipped with various devices for beam diagnostics.

\section{ACKNOWLEDGMENTS}

We would like to acknowledge all members of the KEK-ATF group for the operation of the damping ring and Dr. S. Yamada and Dr. S. Iwata for their continuous encouragement. For the design of the Compton chamber, we express our thanks for close cooperation with Dr. I. Ben-Zvi and Dr. I. Pogorelsky at BNL-ATF and Dr. A. Tsunemi at the Sumitomo Heavy Industries, Ltd. This research was partially supported by a Research Fund of KEK for Cooperative Developments, a Grant-in-Aid for Scientific Research [(B)11554010, (A)11694092], and a research program of Japan/U.S. Cooperation in the Field of High-Energy Physics.

[1] JLC Group, KEK Report No. 92-16, 1992.

[2] JLC Study Group, KEK Report No. 97-1, 1997.

[3] T. Omori, in Proceedings of the 1st ACFA Workshop on Physics and Detector at the Linear Collider, Beijing, China, 1998, KEK Proceedings No. 99-12 (KEK, Tsukuba, 1999), p. 161.

[4] T. Okugi, Y. Kurihara, M. Chiba, A. Endo, R. Hamatsu, T. Hirose, T. Kumita, T. Omori, Y. Takeuchi, and M. Yoshioka, Jpn. J. Appl. Phys. 35, 3677 (1996).

[5] K. Flöttmann, DESY Report No. 95-064, 1995.

[6] K. Hikasa, Phys. Rev. D 33, 3203 (1986).

[7] T. Hirose, in Proceedings of the International Workshop on Physics and Experiments with Linear Colliders,
Morioka-Appi, Japan, 1995 (World Scientific, Singapore, 1996), p. 748.

[8] K. Dobashi, T. Hirose, T. Kumita, Y. Kurihara, T. Muto, T. Omori, T. Okugi, K. Sugiyama, and J. Urakawa, Nucl. Instrum. Methods Phys. Res., Sect. A 437, 169 (1999).

[9] T. Hirose, K. Dobashi, Y. Kurihara, T. Muto, T. Omori, T. Okugi, I. Sakai, J. Urakawa, and M. Washio, Nucl. Instrum. Methods Phys. Res., Sect. A 455, 15 (2000).

[10] T. Omori, in Proceedings of the International Conference on LASER '99, Quebec, Canada (STS Press, McLean, VA, 2000), p. 273.

[11] S. Kashiwagi, M. Washio, T. Kobuki, R. Kuroda, I. Ben-Zvi, I.V. Pogorelsky, K. Kushe, J. Skaritka, V. Yakimenko, X. J. Wang, T. Hirose, T. Muto, K. Dobashi, J. Urakawa, T. Omori, T. Okugi, A. Tsunemi, D. Cline, T. Liu, and P. He, Nucl. Instrum. Methods Phys. Res., Sect. A 455, 36 (2000).

[12] I. Pogorelsky, I. Ben-Zvi, T. Hirose, S. Kashiwagi, V. Yakimenko, K. Kusche, P. Siddons, J. Skaritka, A. Tsunemi, T. Omori, J. Urakawa, M. Washio, and T. Okugi, Phys. Rev. ST Accel. Beams 3, 090702 (2000).

[13] T. Okugi, T. Hirose, H. Hayano, S. Kamada, K. Kuroda, N. Naito, K. Oide, K. Takata, S. Takeda, N. Terunuma, N. Toge, J. Urakawa, S. Kashiwagi, M. Takano, D. McCormick, M. Minty, M. Ross, M. Woodley, F. Zimmermann, and J. Corlett, Phys. Rev. ST Accel. Beams 2, 022801 (1999).

[14] K. Kubo et al., Phys. Rev. Lett. 88, 194801 (2002).

[15] SAD is a computer program for accelerator design, http:// acc-physics.kek.jp/SAD/sad.html

[16] GEANT detector description and simulation tool, CERN Program Library Long Writeup W5013, 1994.

[17] GLAD (General Laser Analysis and Design) is an optical software package produced by Applied Optics Research, AOR, http://www.optima-research.com/Software/Laser/ GLAD/index.htm

[18] I. Sakai, K. Dobashi, M. Fukuda, A. Higurashi, T. Hirose, T. Iimura, Y. Kurihara, T. Okugi, T. Omori, J. Urakawa, and M. Washio, KEK Preprint No. 2001-115, 2001.

[19] M. Fukuda, T. Hirose, Y. Kurihara, A. Ohashi, T. Okugi, T. Omori, J. Urakawa, M. Washio, and I. Yamazaki, in Proceedings of the Joint 28th Advanced Beam Dynamics \& Advanced \& Novel Accelerators Workshop on Quantum Aspects of Beam Physics-and Other Critical Issues of Beams in Physics and AstrophysicsHigashi-Hiroshima, 2003 (Hiroshima University, Higashi-Hiroshima, 2003).

[20] M. Fukuda, T. Aoki, K. Dobashi, T. Hirose, T. Iimura, Y. Kurihara, T. Okugi, T. Omori, I. Sakai, J. Urakawa, and M. Washio, KEK Preprint No. 2003-5, 2003 [Phys. Rev. Lett. (to be published)].

[21] G. Culligan, S. G. F. Frank, J. R. Holt, J. C. Kluyver, and T. Massam, Nature (London) 180, 751 (1957).

[22] M. Goldhaber, L. Grodzins, and A.W. Sunyar, Phys. Rev. 109, 1015 (1958).

[23] P. C. Macq, K. M. Crowe, and R. P. Haddock, Phys. Rev. 112, 2061 (1958). 